
201524060143

作者姓名 Ibrahim Abdulai Sawaneh

指导教师 Professor Jian-Ping Li 
分类号

密级

$\mathrm{UDC}^{\text {注 } 1}$

$$
\text { 不丮 }
$$<smiles>CCC1(C)CCCC1(C)C</smiles>

护

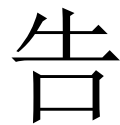

DWT Based Image Compression for Health Systems (题名和副题名)

\section{Ibrahim Abdulai Sawaneh}

（作者姓名）

指导教师

Professor Jian-Ping Li

电子科技大学 成 都

（姓名、职称、单位名称）

申请学位级别 硕士 学科专业 Computer Science and Technology

提交论文日期 2017.04 论文答辩日期—2017.05

学位授予单位和日期电子科技大学 2017 年 6 月

答辩委员会主席

评阅人

注 1: 注明《国际十进分类法 UDC》的类号。 


\title{
DWT Based Image Compression for Health Systems
}

\author{
A Master Report Submitted to \\ University of Electronic Science and Technology of China
}

Discipline:

Computer Science and Technology

Author:

Ibrahim Abdulai Sawaneh

Supervisor:

Professor Jian-Ping Li

School: Computer Science and Engineering of UESTC 


\section{独创性声明}

本人声明所呈交的学位论文是本人在导师指导下进行的研究工作 及取得的研究成果。据我所知, 除了文中特别加以标注和致谢的地方 外, 论文中不包含其他人已经发表或撰写过的研究成果, 也不包含为 获得电子科技大学或其它教育机构的学位或证书而使用过的材料。与 我一同工作的同志对本研究所做的任何贡献均已在论文中作了明确的 说明并表示谢意。

作者签名:

日期: 年 月 $日$

\section{论文使用授权}

本学位论文作者完全了解电子科技大学有关保留、使用学位论文 的规定，有权保留并向国家有关部门或机构送交论文的复印件和磁盘， 允许论文被查阅和借阅。本人授权电子科技大学可以将学位论文的全 部或部分内容编入有关数据库进行检索, 可以采用影印、缩印或扫描 等复制手段保存、汇编学位论文。

（保密的学位论文在解密后应遵守此规定）

作者签名: 导师签名

日期: 年 月 日 


\section{摘 要}

当涉及处理数据量大的患者记录时, 呼吁加强现有医疗行业。巨大的文件包 含大量的副本。因此, 压缩的理想发挥作用。图像数据压缩消除了增加存储空间 和传输带宽的几余副本 (多个不必要的副本)。图像数据压缩是关键的, 因为它 有助于减少图像文件的大小, 并通过多种小波分析方法在互联网上加速文件传输 速率, 而不会损失传输的医学图像数据。

因此, 本报告提出了使用离散小波变换（DWT）, 傅里叶变换（FT）和快速傅 里叶变换的提出的方案进行医疗系统的数据压缩实现, 具有压缩和恢复医学图像 数据而没有数据丢失的能力。医疗图像如人类心脏和大脑需要快速传输, 以获得 可靠和高效的结果。使用具有最佳重建质量的 DWT 大大提高了压缩。

通过有效的数据压缩技术, 可以实现通过大数据进行健康监测的通信技术创 新。我们的实验实验表明, 使用 Haar 小波参数确定 MSE 和 PSNR 解决了我们的目 标。还部署了许多成像技术, 以进一步确定 DWT 方法的效率, 如图像压缩和图像 去噪。拟议的医学图像压缩效果非常好。

通过采用压缩程序缩小存储空间, 降低传输速率, 并限制卫生监控系统中的 大量能源消耗, 必须减少数据集的大小。这项工作的动机是实施压缩方法来修改 传统医疗平台, 降低文件大小, 降低运营成本。图像压缩旨在从与非零系数关系 中已经认为必要的广泛较小的估计重建图像。合理地, 较少选择良好的解释足以 使新样本与源图像完全一致。我们看 DWT 实现我们的压缩方法。

关键词: 离散小波变换 (DWT), 哈尔变换, 图像压缩医学图像。 


\begin{abstract}
There are calls for enhancing present healthcare sectors when it comes to handling huge data size of patients' records. The huge files contain lots of duplicate copies. Therefore, the ideal of compression comes into play. Image data compression removes redundant copies (multiple unnecessary copies) that increase the storage space and transmission bandwidth. Image data compression is pivotal as it helps reduce image file size and speeds up file transmission rate over the internet through multiple wavelet analytics methods without loss in the transmitted medical image data.

Therefore this report presents data compression implementation for healthcare systems using a proposed scheme of discrete wavelet transform (DWT), Fourier transform (FT) and Fast Fourier transform with capacity of compressing and recovering medical image data without data loss. Healthcare images such as those of human heart and brain need fast transmission for reliable and efficient result. Using DWT which has optimal reconstruction quality greatly improves compression.

A representation of enabling innovations in communication technologies with big data for health monitoring is achievable through effective data compression techniques. Our experimental implementation shows that using Haar wavelet with parametric determination of MSE and PSNR solve our aims. Many imaging techniques were also deployed to further ascertain DWT method's efficiency such as image compression and image de-noising. The proposed compression of medical image was excellent.

It is essential to reduce the size of data sets by employing compression procedures to shrink storage space, reduce transmission rate, and limit massive energy usage in health monitoring systems. The motivation for this work was to implement compression method to modify traditional healthcare platform to lower file size, and reduce cost of operation. Image compression aims at reconstructing images from extensively lesser estimations than were already thought necessary in relations with non-zero coefficients. Rationally, fewer well-chosen interpretations is adequate to reproduce the new sample exactly as the source image. We look at DWT to implement our compression method.
\end{abstract}

Keywords: Discrete Wavelet Transform (DWT), Haar Transform, Image Compression Medical Image. 


\section{Contents}

Chapter 1 Introduction ..................................................................................................... 1

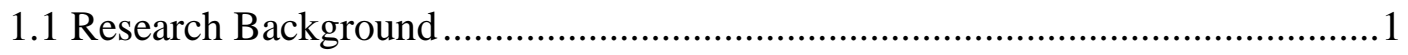

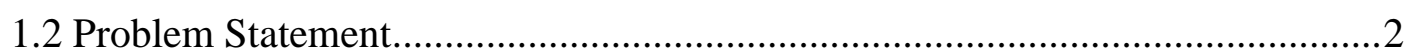

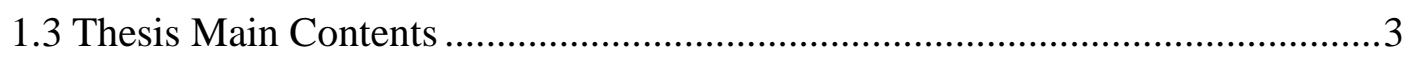

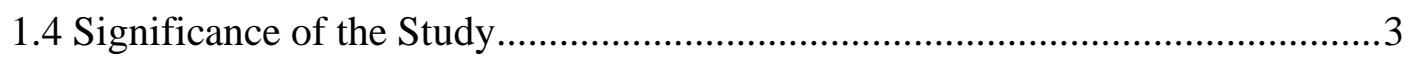

1.5 Thesis Main Aims and Objectives ................................................................4

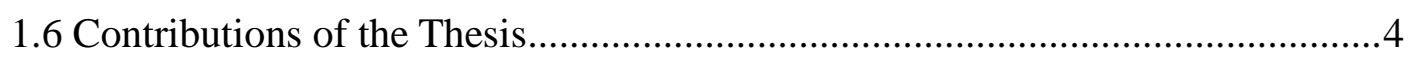

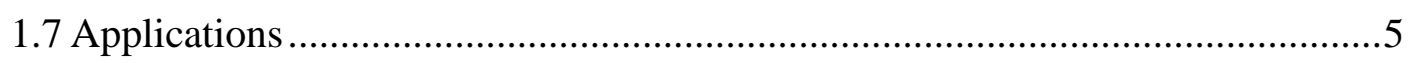

1.7.1 Magnetic Resonance Imaging (MRI) .............................................. 5

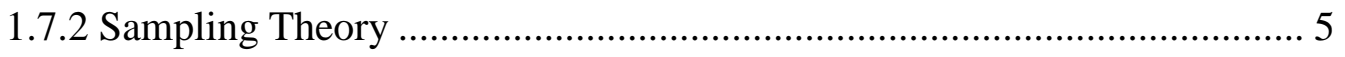

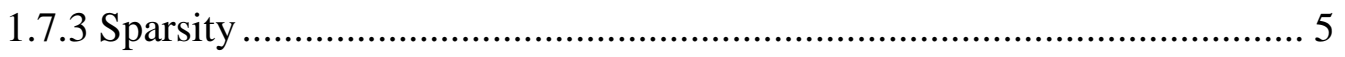

1.7.4 Wearable Wireless Body Area Network (WWBAN) ............................... 6

1.7.5 Wearable Smart Shirt Application ........................................................ 7

1.7.6 Health Monitoring System ................................................................ 7

1.7.7 Trend in Health Monitoring Systems ..................................................... 10

1.8 Health Monitoring Systems Challenges .......................................................... 11

1.8.1 Sensitivity of Sensors ...................................................................... 11

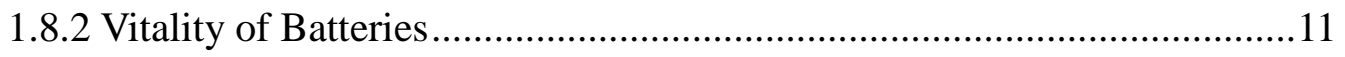

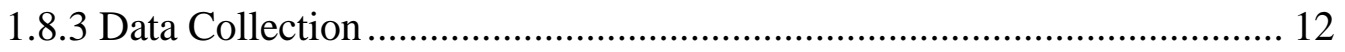

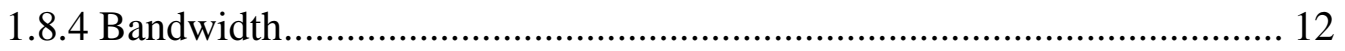

1.9 Autonomous Challenges for health Monitoring Systems ................................12

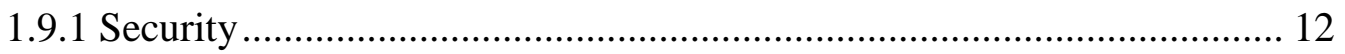

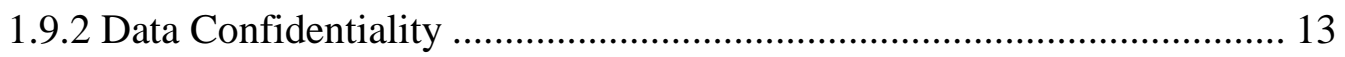

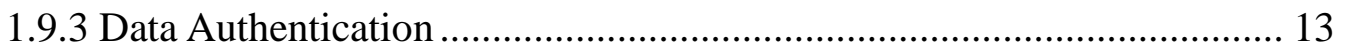

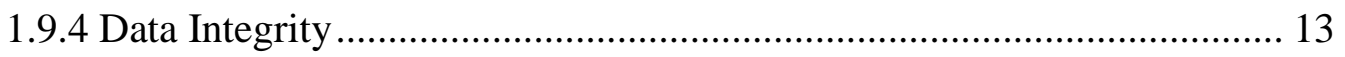

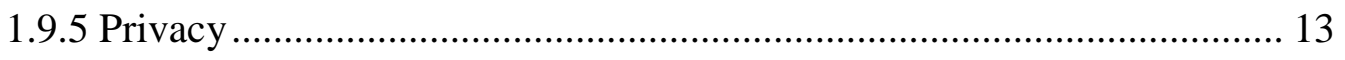

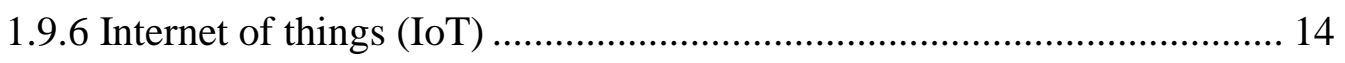

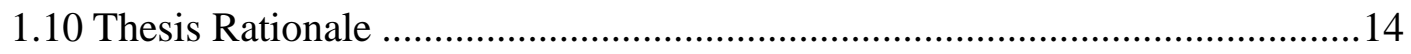

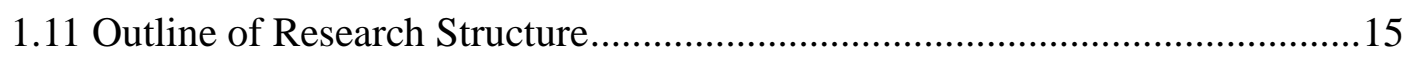

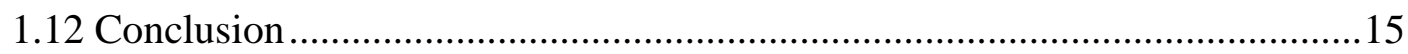




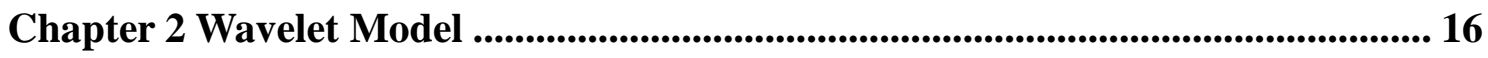

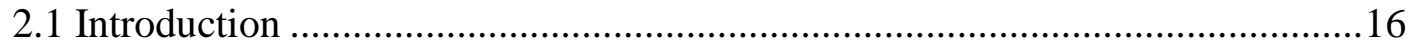

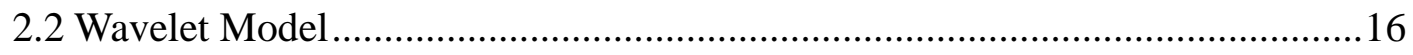

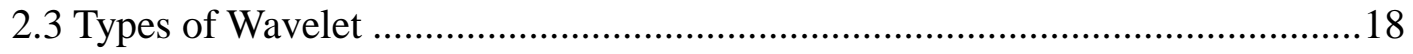

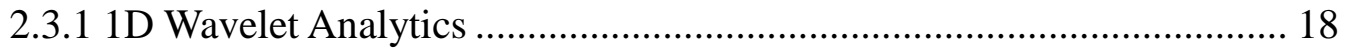

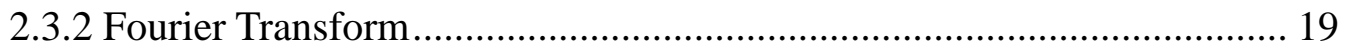

2.3.3 Power Spectral Density (PSD) Estimation .......................................... 20

2.3.4 Discrete Wavelet Transform (DWT) .................................................. 21

2.3.5 Discrete Wavelet Series .......................................................................... 22

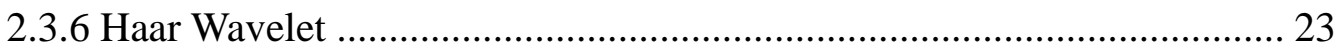

2.3.7 Multiresolution ......................................... Error! Bookmark not defined.

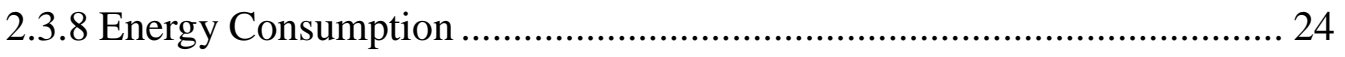

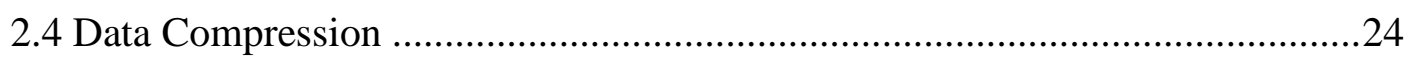

2.4.1 Data Compression Advantages ......................................................... 25

2.4.2 Data Compression Disadvantages ...................................................... 25

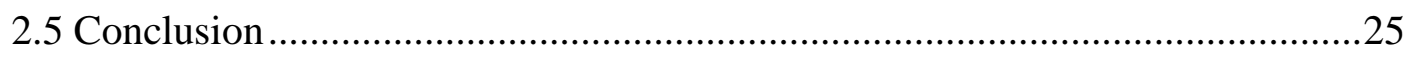

Chapter 3 Methodology............................................................................................. 27

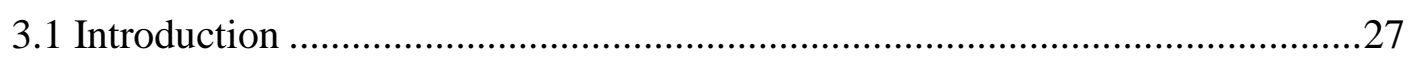

3.2 Medical Image Compression Overview ....................................................22

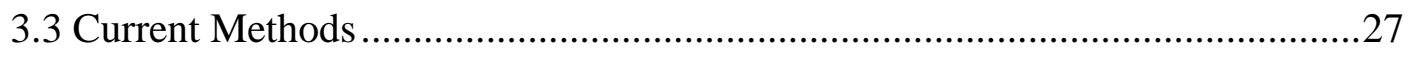

3.3.1 Discrete Cosine Transform (DCT) Based Coding ............................... 27

3.3.2 Discrete Wavelet Transform (DWT) Based Coding .............................. 28

3.3.3 Huffman Based Coding and Decoding ................................................. 28

3.4 Proposed Compression Method Implementation with (DWT) ........................29

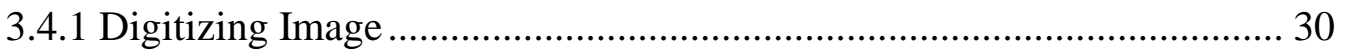

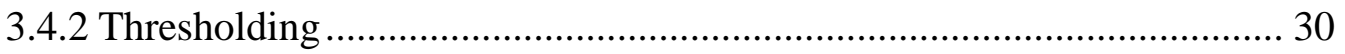

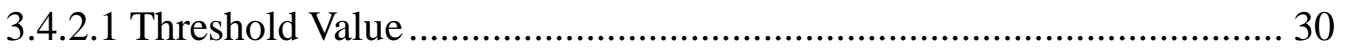

3.4.2.2 Applying thresholding to a value ........................................................ 31

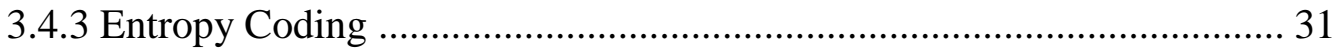

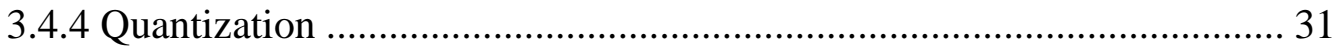

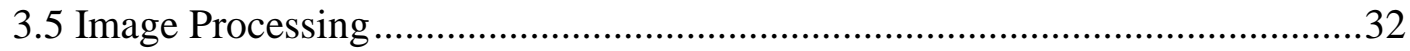

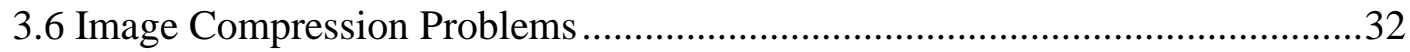

3.6.1 Compression Method Used ….............................................................. 32 


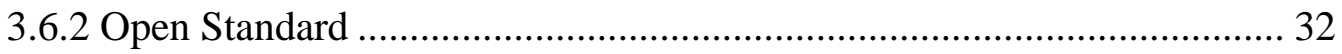

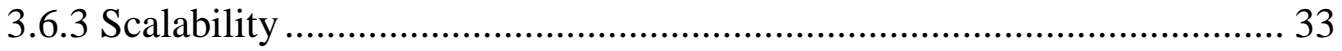

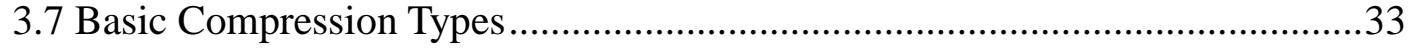

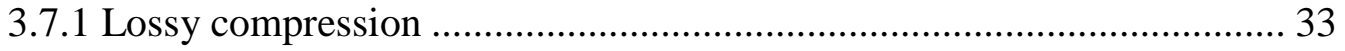

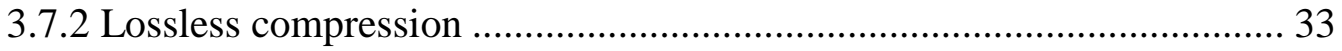

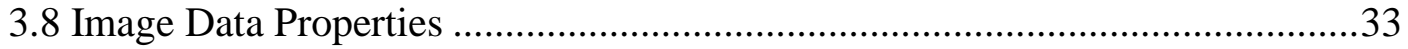

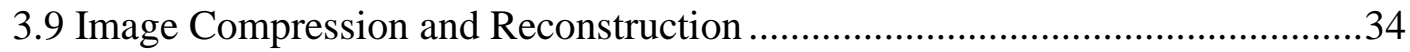

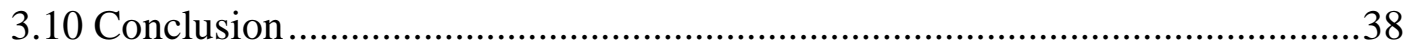

Chapter 4 Implementation and Testing DWT-based Image Compression in Matlab

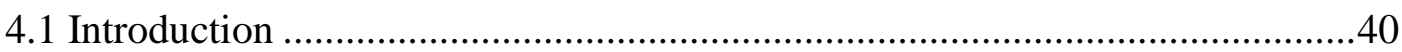

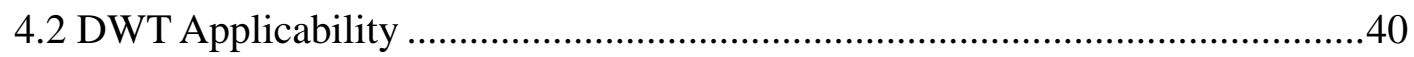

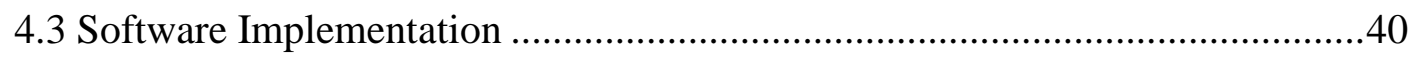

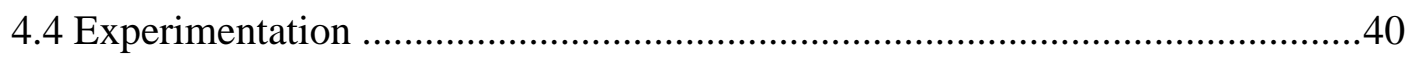

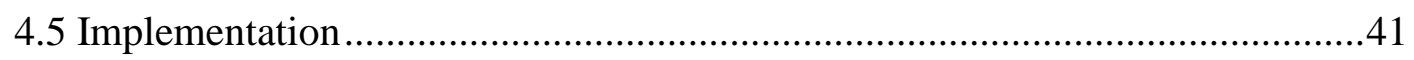

4.5.1 FFT Implementation Setup ............................................................ 41

4.5.2 Energy Loss or Gain Implementation Setup....................................... 42

4.5.3 Experimentation Result for Image Compression.................................. 43

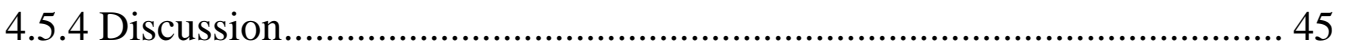

4.6 Image De-noising in Wavelet ..............................................................45

4.6.1 Compression in Wavelet 2D .......................................................... 45

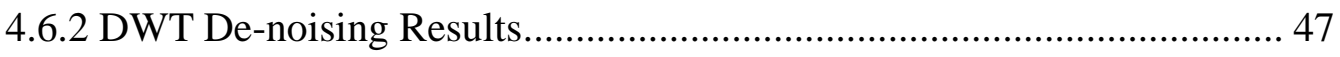

4.7 More DWT Compression Implementation in Matlab......................................48

4.7.1 Experimental Result Discussion for figure 4-7 .................................. 48

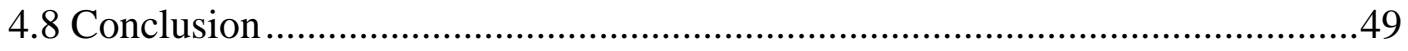

Chapter 5 Conclusions and Future Recommendations................................................ 50

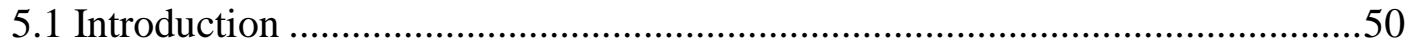

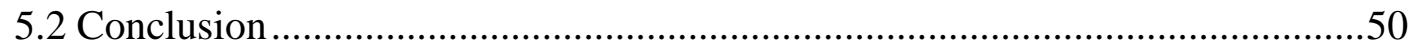

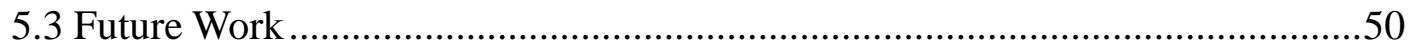

Acknowledgement ................................................................................................................. 51

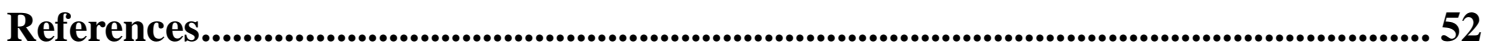

Appendix I: Calculation of MSE and PSNR in Matlab ......................................... 55

Appendix II: Fast Fourier Transform implementation code...................................56 


\section{List of Figures}

Figure 1-1 Wearable wireless body area network architecture .................................... 9

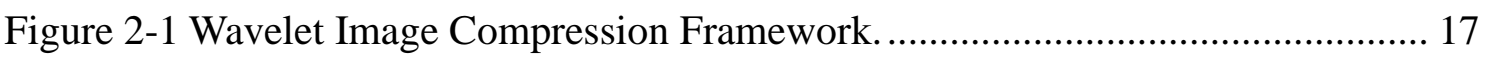

Figure 2-2 Approximation and Detail Framework ................................................... 18

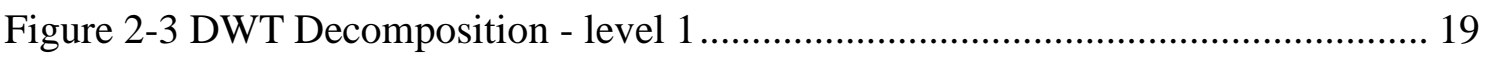

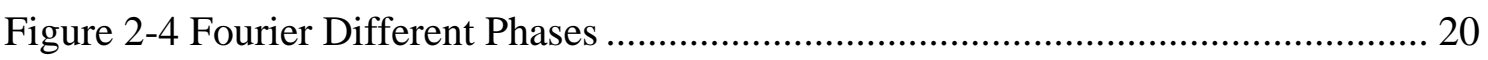

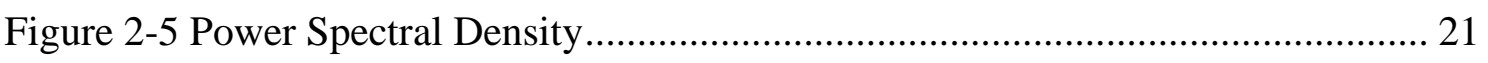

Figure 2-6 Wavelet Detail and Coefficients Sub-signal framework .............................. 22

Figure 2-7 Data Compression Structural Model.......................................................... 24

Figure 3-1 DCT Image Compression Architecture .................................................... 28

Figure 3-2 Wavelet Transform compression Framework ........................................... 29

Figure 3-3 Wavelet Image compression and decompression ..................................... 35

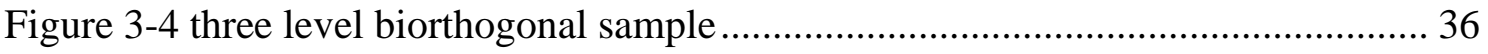

Figure 3-5 MSE, PSNR and CR values of different performance indexes for Brain ..... 37

Figure 3-6 MSE, PSNR and CR values of different performance indexes for Heart ..... 38

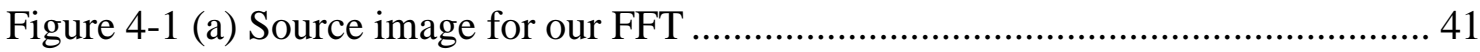

Figure 4-1 (b) Converted FFT image black and white ................................................ 41

Figure 4-1 (C) Computed FFT of our image using fft2 ….......................................... 42

Figure 4-1 (d) Zeroing small coefficients and inverse transform ................................ 42

Figure 4-2 Illustration of Energy Loss or Gain .......................................................... 43

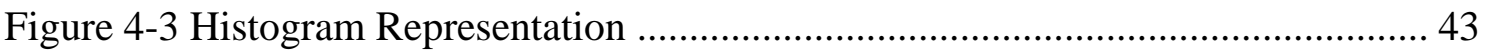

Figure 4-4 Histogram Representation for DWT and other compression frameworks ... 44

Figure 4-5 Histogram Representation for DWT compression comparison ................... 45

Figure 4-6 (a) DWT de-noised medical image.............................................................. 46

Figure 4-6 (b) four level of the de-noised medical image. .............................................. 46

Figure 4-6 () More level details of the de-noised medical image. ................................. 47

Figure 4-7 Experimental Result done in Matlab .......................................................... 48 


\section{List of Tables}

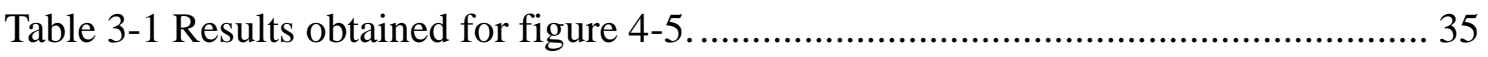

Table 3-2 MSE, PSNR and CR values of different performance indexes for Brain ...... 37

Table 3-3 MSE, PSNR and CR values of different performance indexes for Heart ...... 38

Table 4-1 Results obtained from different threshold standards with proposed

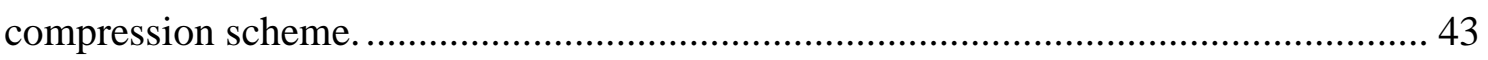

Table 4-2 Showing comparison between DWT and other compression frameworks .... 44

Table 4-3 Comparison of DWT compression schemes .............................................. 44

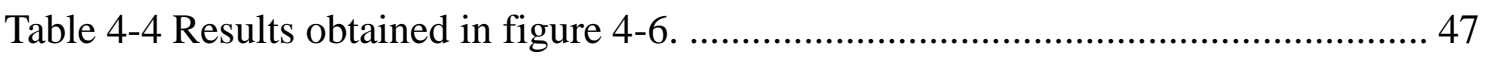




\section{List of Glossary}

$\begin{array}{ll}\text { DWT } & \text { Discrete Wavelet Transform } \\ \text { DCT } & \text { Discrete Cosine Transform } \\ \text { FFT } & \text { Fast Fourier Transform } \\ \text { CR } & \text { Compression Ratio } \\ \text { PSNR } & \text { Peak Signal to Noise Ratio } \\ \text { MSE } & \text { Mean Square Error } \\ \text { FT } & \text { Fourier Transform } \\ \text { WT } & \text { Wavelet Transform } \\ \text { DFT } & \text { Discrete Fourier Transform } \\ \text { ADC } & \text { Analog - to- Digital Converter } \\ \text { PDA } & \text { Personal Digital assistance } \\ \text { PC } & \text { Personal Computer } \\ \text { IoT } & \text { Internet of Things } \\ \text { ITU } & \text { The International Telecommunication Union } \\ \text { CERP-IoT } & \text { Cluster of European Research Projects - Internet of Things } \\ \text { ICT } & \text { Information and Telecommunication Technology } \\ \text { CPU } & \text { Central Processing Unit } \\ \text { MRI } & \text { Magnetic Resonance Imaging } \\ \text { NASA } & \text { The National Aeronautics and Space Administration } \\ \text { DES } & \text { Data Encryption Standard } \\ \text { ADES } & \text { Advance Data Encryption Standard } \\ \text { ECC } & \text { Elliptical Curve Cryptography } \\ \text { MAC } & \text { Message Authentication Code } \\ \text { PERS } & \text { Personal Emergency Response Systems } \\ \text { RAM } & \text { Random Access Memory } \\ \text { FBI } & \text { Federal Bureau of Investigation } \\ \text { CIA } & \text { Central Intelligence Agency } \\ \text { MI6 } & \text { Britain's Secret Intelligence Service } \\ \text { KGB } & \text { Komitet gosudarstvennoy bezopasnosti (Russian Secret Service } \\ \text { BBC } & \text { Bnstitution) } \\ \text { CCTV } & \text { Closed-Circuit Television } \\ \text { VOA } & \text { SLBS }\end{array}$




\section{Chapter 1 Introduction}

\subsection{Research Background}

Patient monitoring in health connected topic is becoming important issues in healthcare domains and beyond. Tele-monitoring is the latest medical modernization which deploys internet technology remotely monitoring patient body's symptoms independently. Health monitoring platform renders effective Medical tools in monitoring of diseases especially ageing populace ${ }^{[1]}$. Patient medical supervision reduces risk infectious diseases spread and also minimize hospitalization cost. More sicknesses are practically found every year, and medical constrains particularly undeveloped countries has rocketed healthcare costs globally. Heath monitoring enhances the predictability of life frightening illnesses, and institutes an alarming mechanism for catastrophic scenarios where prompt treatments are delivered saving death rate ${ }^{[2]}$.

The high demand and speedy surge in global population with diverse medical needs because of man's interference with nature, has triggered an alarm by healthcare experts to introduce real and resourceful alternative healthcare solutions for earth's population. Several establishments such as hospital-care, prison-care, space-care, and clinic-care ${ }^{[3]}$ exploits health monitoring platform to relentlessly monitor in real-time. This eases hospitalization time and cost, augmenting user's day-by-day schedule while been monitored by healthcare experts.

This prospect has built another exploration in some research areas to supplement customary healthcare which have ended up incapable to thoroughly check patients' vital body records remotely. Health monitoring systems removes limitation on mobility with improved quality for healthcare sector ${ }^{[4]}$.

The use DWT and similar technique greatly decrease size of medical gadgets through compression and make its implementation extra demanding for health monitoring applications. This is because medical monitoring applications like wearable or implantable biosensor require portability and comfort to user. The thorough checking of vital body symptoms in area with limited doctor's facilities and healthcare experts particularly when patient is restrained help in early finding of ailments. Therefore, within hospital and remote treatment institutions are enormously operational for 
nonstop checking of essential body signs. Health monitoring scheme of lately have energetically made space into medical sectors where remote sensors advances are been actualized in data compression system designed for medicinal purpose.

Health monitoring platform urges patient to completely watch changes of essential body parameters, and get advice from medical experts consistently in attaining optimal healthcare standard. In this way, utilizing health monitoring platform viably builds quality, proficiency and viability for the proposed structure in observing patient's wellbeing with better and optimal framework.

The ultimate drive of this work is to organize low compression technique to tackle the huge data size and high transmission bandwidth for medical data image. Image data compression is aims at reconstructing images and signals from comprehensively fewer calculations. And rationally, less well-chosen interpretation is suitable to reproduce the new measurement exactly as the true signal. Having little understanding about signal's sparsity, the original image is reproduced with even smaller samples. Compressionbased algorithms are built upon this assumption. Most biomedical records lack sparsity in either time or transform domains with redundancies. The implementation for health monitoring systems using DWT, FT and FFT result to effective healthcare services as the collected medical data attained are analyzed, and stored with lesser storage room and subsequently reduces the transmission rate, and allow our scheme to use mobile phone which has limited storage facility.

\subsection{Problem Statement}

As we know health monitoring over lingering time frame result to huge data size. Medicare data for transmission on the internet should be compress to reduce file size, lower upload time, and transmission interval. Image compression plays pivotal role for health monitoring system and widely implemented across engineering fields. It is huge challenge to develop resourceful compression scheme to resolve huge data problem, storage and transmission interval. Also transmitting unprocessed digital data pose difficulty at the receiving PC and subsequently result in high cost of operation. Medical data are sensitive life threatening information which means during transmission, no detail records should be lost.

The numerous stated and unstated problems can be accomplished by using DWT which has higher data compression and reconstruction superiority in Matlab. The 
proposed model provides effective answers for health monitoring systems using DWT, Fourier transform, Fast Fourier transform and Haar wavelet to decompress and reconstruct healthcare image signals without information loss to obtain the research objectives (reduce data size, reduce transmission interval and requires minimal energy use).

\subsection{Thesis Main Contents}

We did an excellent work in the field of Wavelet for image compression in health monitoring systems. We were tasked how to remove duplicate copies in image file without affecting image quality, and also how to reconstruct the image signal without data loss ${ }^{[5]}$. Therefore, we proposed a DWT-based Image Compression method to overcome challenges posed by massive data sizes. Our implementation developed is efficient and it uses PSNR and MSE achieves superior compressibility. This removes difficulties posed by transfer of large file sizes in medical image processing and gives better accuracy in compression and reconstruction.

\subsection{Significance of the Study}

Lately, numerous technological innovation driven algorithms for medicare image compression has been established. The continuous growth in healthcare services seen in health monitoring systems, wearable healthcare system, wireless wearable body area network (WWBAN), and e-therapy consultancy demand efficient medicare image compressibility and reconstruction. Healthcare professionals monitoring patients' conditions are face with difficulty of receiving patient data as required due to data transmission error attributing to huge file size, and sometimes loss of patient data. An efficient image data compression platform that removes redundant portions and maintains the file vital content is needed to solve big data size. This brings into play for Lossless compression [6]-[7], referred to entropy coding decompose and reconstruct medical images excellently without of loss of image detail, but has compression ratio issue. Lossy image compression platform is less effective in medicare image compression because of data loss attributed to it, but possess high compression ratio than Lossless compression.

Looking at which compression schemes that provides the optimum compression ratio for healthcare images are essential feature of our research. Our proposed 
implementation is removing duplicate copies in medicare images to provide lesser storage space on storage devices (HD drive and others) and enhance optimal transmission time and also reduce cost of operation. This lead to small file size and grants multiple image signals to be store on a given disk ${ }^{[8]-[9]}$. To achieve outstanding and cost-effective compression method is to use Lossless compression that shows outstanding medicare image compression with high quality. Our proposed work is to implement a DWT image compression in health monitoring systems are attainable.

\subsection{Thesis Main Aims and Objectives}

The main aims and objectives of this report are:

1. To implements DWT and Fourier transform to compress well-being images with superior performance.

2. To decrease storage room for medical records.

3. To lower transmission interval and retrieval for healthcare data.

4. Maintain file quality before and after transmission.

5. Use MSE, CR and PSNR in Matlab to show the effectiveness and accuracy of the implementation.

6. Provide affordable healthcare cost and diminish long hospital stay.

7. Uses different wavelet types such as Fourier transform, Fast Fourier transform and Haar to compare their effectiveness.

\subsection{Contributions of the Thesis}

Electronic well-being supervisions are conceivable via intuitive monitoring platform with instantaneous remote communications amongst users and caregivers ${ }^{[10]}$. DWT, and Fourier Transform algorithm features higher superiority compression aptitude to undetermined matrix analysis. If applied accurately, will achieve dire medical needs (affordability and mobility) in health monitoring sector. Health monitoring of fundamental well-being indicators are crucial in solving numerous drawbacks (gadget cost, lightweight medical sensors) in health platform. The demand of man's continuous misuse of universe's resources in exploration of knowledge has created devastating consequence (climate change) causing various chronicle illnesses. Meanwhile, the rocketry healthcare expenditure and incremental growth in human 
population have given governments globally taking proactive stance to render its citizens with inexpensive, and efficient healthcare platform that constantly monitor people's health status at anyplace, and anytime via health monitoring system. Redesigning existing medical applications that counter sicknesses in a readily proactive manner are pivotal for nonstop patient monitoring that is effective and affordable. The huge patient data amassed are compressed via DWT, FT, FFT and Haar in Matlab.

\subsection{Applications}

This subsection vividly details few problems affecting customary compression scheme (lossy compression). Many innovative compression domains predominately produced optimum effects in medical field such as Magnetic Resonance Imaging (MRI), Medical Image Processing, Signal Processing, Sampling Theory, Error Correction, and Data Compression

\subsubsection{Magnetic Resonance Imaging (MRI)}

An anticipating utilization of medicare image to successfully compressed and recover signals. MRI essentially medical innovation uses pictorial representation. Shannon Nyquist theorem consumes more energy, cost, and time in obtaining medical imagery in quantifiable circumstances. This is evident in human heart requiring lesser treatment time with higher resolution for medicare images within few minutes. Nevertheless, it has few drawbacks in healthcare domain like efficiency rate and excessive analysis procedures.

\subsubsection{Sampling Theory}

Recovering continuous-time signal from discrete data sample is instrumental for numerous innovative researches. It efficiently recovers real continuous-time signal measurements. Such uses are seeing in image processing, biomedical sensor innovation, compressibility rate.

\subsubsection{Sparsity}

Sparsity suggests that most components measurement matrices are zero or closed to zero. Sparse approximation is applied in image processing, biological science, documentation, and auditory assessment for representation and compressibility. 
Compression, a component of sparse approximation in auditory assessment, atmospheric resonance, etc. This is evident by Adiloglu et al ${ }^{[11]}$ matching pursuit for time-frequency components. Scholler and Purwins approximation of drum noise ${ }^{[12]}$. Adiloglu et al in area of biomedical, it is cost-effectively viable and effective to compressed physiological records in DNA miniaturized scale cluster test, where small quantity of aggregated amount of genes assessment are differentially negligible records with high quality before communicated.

\subsubsection{Wearable Wireless Body Area Network (WWBAN)}

WWBAN, a pivotal infrastructure for ubiquitous and nonstop monitoring through WSN infrastructure has tremendously improved life standard, helps medical experts to wirelessly monitor patient health conditions with a likelihood of success. Reduce prolong hospitalization and congestion in hospitals, initiate early discovery of illnesses etc. Smart shirt comprises of several wearable sensors used to examine patients' vital body parameters.

The persistent patient monitoring routine for diagnostic procedures improves therapy consequence of medicines. Pedometers are extensively used in healthcare and facilitate patients having complete control of their well-being status ${ }^{[13]}$. Biomedical sensors convert body signals including to electrical samples and communicate to network coordinator. NASA ${ }^{[14]}$ is designing wearable covering to manage heartbeat, blood pulse and other biological records for astronauts. Numerous wearable sensor kits are lately designed such as Lifeshirt by Vivo metrics, body monitoring system ${ }^{[15]}$.

There is great achievement for sensor that monitors physical property compare to wearable chemical sensor in ubiquitous examination for bodily fluids. Chemical sensor has many setbacks such as sample gathering and delivery, wearability, sensor calibration, and safety concerns.

\subsubsection{Future of WWBAN System in Health Monitoring Systems}

For better understanding and future implementation of WWBANs in healthcare infrastructure, it is noteworthy to browse the following important standardized improvements;

$\checkmark$ Excellent compression technique subsequently reduces wearable sensor size, cost-effective and optimizes energy use which increases sensor battery. 
$\checkmark$ Patient records must be encrypted to fulfill the basic security requirements as it's entrain vital health data, if release to third party will create traumatic and devastating consequence to patient well-being, (HIV and aids records).

$\checkmark$ WBASN coordinates numerous medical sensors with varying frequencies incorporating adaptive telecommunication protocols.

$\checkmark$ Ensuring instantaneous wireless monitoring of patients' body measurements. Several gateway gadgets must be implemented complementing archaic healthcare system.

$\checkmark$ A universal accepted standard in sensor design, data gathering and storage.

$\checkmark$ Flexibility and incorporation for wearable sensor in standalone WWBANs implementation to facilitate recording of medical data while on-line and off-line to detect any strange condition in patients.

\subsubsection{Wearable Smart Shirt Application}

Several wearable health monitoring schemes designed recently has skill to endlessly examine well-being conditions particularly ageing and disable. An example is the wearable infrastructure known as 'Smart Vest' which examines many body measurements; e.g., electrocardiograms, heart pulse, blood rate, and respiratory activity.

E- Shirt examines ECG in real-time implemented. The shirt is embedded with sensors designed for ubiquitous examination, and the conducting fabric acts as electrodes to collect human body signs. The processed ECG details are communicated to ad-hoc network (IEEE 802.15.16) for distant supervision. Medical monitoring gadgets as in wrist provide immediate response for high-risk cardiac patients to examine physiological measurements.

\subsubsection{Health Monitoring System}

With swift acceleration in ageing populace combines with exponential rise in healthcare expenditure have triggered innovation driven enhancements to current medical system. Administering cure to patient wired or remotely without been hospitalized is now possible. A blend of schemes is being developed recently for realtime feedback monitoring. Moreover, modern advance in electronics and wireless tools have proficiently improved tele-monitoring. These sensors gather crucial signals about body's state, and thus facilitate the presentation of new sort of networks among called 
health monitoring systems. Health monitoring systems are system of hubs capacitating patient records for detail examination. Importantly, data accrued from different sensors are conveyed to healthcare servers for further examination and storage (big data). Therefore, engaging wired link will be awkward and results to enormous cash for operation. Contradictory, implementing wireless innovations diminishes cost and size. Health tele-monitoring platform is system with successful, solid, secure, reliable, and effective framework. Portability enhances mobility, and necessitates daily routine without any hindrance which lacks in traditional healthcare system.

Adopting tele-monitoring scheme for undeveloped nation's especially African countries, Health monitoring plays greater role for ageing and less privilege in isolated communities with limited healthcare amenities and/or unskilled healthcare experts. It is envisaged with support of modern healthcare applications; e.g. WWBANs, telemedicine, e-Health, tele-pharmacy, etc. through tele-monitoring platform. Health monitoring removes constrains on disadvantaged healthcare domain ${ }^{[16]}$. Moreover, the deployment hinges on resourceful energy along with improve information and telecommunication technology (ICT) utilization which permits instantaneous transmission of medicare records using internet of things.

Wearable health tele-monitoring platform is use of ICT into smart clothing and physiological sensors to constantly check well-being condition far from medical facility. It helps solve elongated hospital stay with reduce cost, and aid moveability for users. Individual nations should be encouraged in financing healthcare rather users and hospitals insuring the financial implications.

General health standards instituted globally in reference to healthcare financing, licensing, and medical education and continuously provide training to increase pervasive adoption in wearable health tele-monitoring system. The essential difference between our system and traditional healthcare is the complete minimization of patient visitation to hospitals and supervising health conditions remotely using internet.

Wireless wearable health tele-monitoring platform is pivotal in changing our health standard in future. Our work highlights high quality, and steadfastness offering flexibility in wearability system, and combination of vast accumulation of wireless networks into existing dedicated medical services. Normally, a sensor or actuator is a miniature gadget embedded into our clothes, e.g. pocket, and convey by people in 
nearness in sacks, wrist watch, bracelets, etc. for monitoring human physiological signs. The high demand for wearable tele-monitoring with improved data compression method, as in DWT has recently surfaced to precisely compute physiological signs with excellent performance, significantly reduces huge data size, high transmission rate, lesser biosensor size enhancing interpretability for medicinal gadgets by users.

Wearable health tele-monitoring system urges patients to completely watch unusual symptoms in body signs, and get medical advice from experts in fulfilling ultimate healthcare standards. Some merits from remotely checking patient condition involves identifying crisis situation, allowing patient to work outside hospital, minimize healthcare cost, enhancing security alarms, and allowing these alarms to be use via internet by healthcare experts administering speedy medication to patient. In this way, deploying wearable health tele-monitoring platform viably builds quality, proficient and viability in checking patient's health state with optimal framework.

The thorough checking of vital body signs with limited healthcare experts particularly when patient is restrained helps detect ailments. Appropriate administering within patient health confine with correct treatment totally lessens fatality. Therefore within hospital and remote treatment centers is great degree operational for nonstop checking of essential body parameters.

Figure 2-1 is a structure for WWBAN consisting of various health monitoring components through internet within wearable health tele-monitoring system.

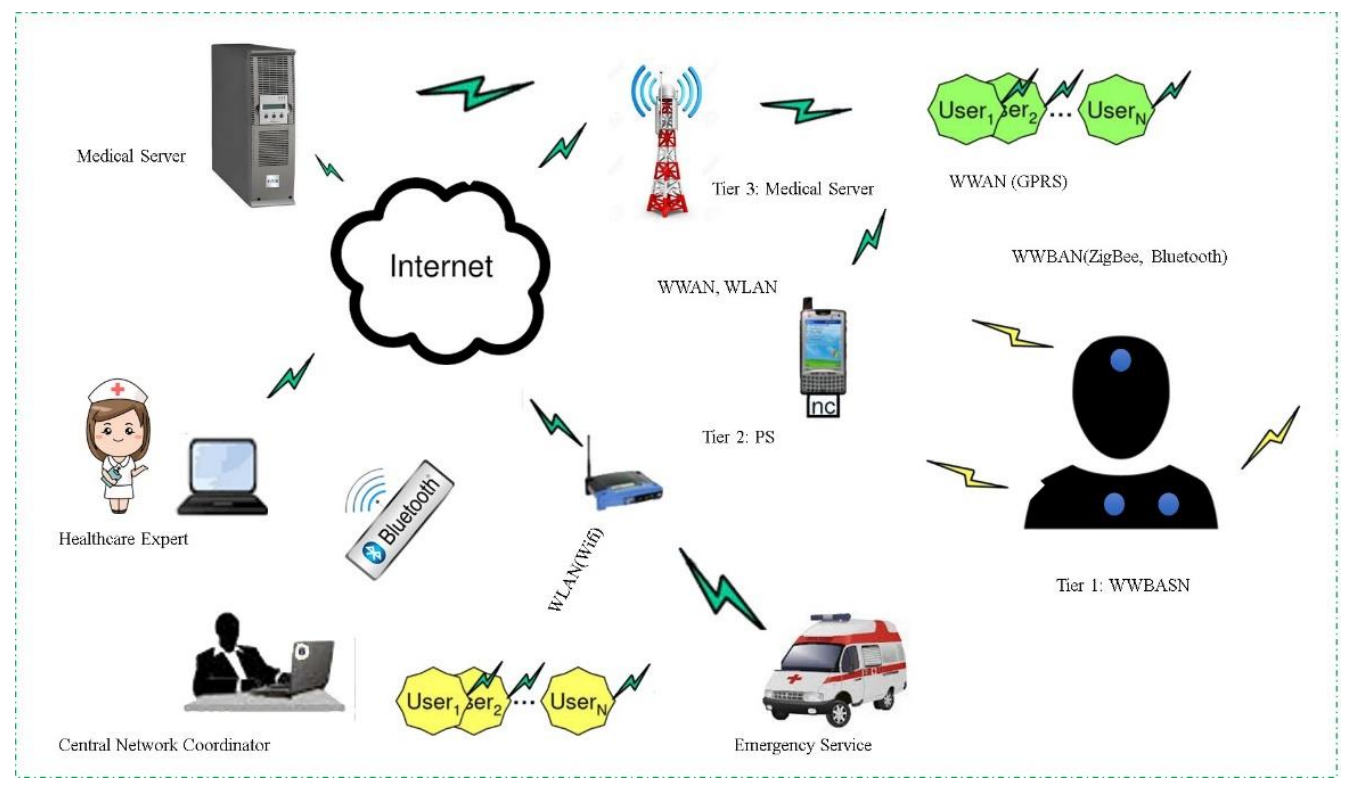

Figure 1-1 Wearable wireless body area network architecture 


\subsubsection{Medical Server/Healthcare Server}

Healthcare server is a processing facility where patient health related electronic data are processed, analyzed, aggregated, stored, and transmitted to medical experts and users when required. It works with numerous wearable sensors implemented by the users and prompt instantaneous remote tele-monitoring of body signals. Healthcare professionals examine, describe, predict and prescribe treatment based on received medical records, especially for emergency situations.

\subsubsection{Personal Server}

Personal server bridges between WWBASN and medical server via wireless communication links. It renders graphical interface or sound interface to user via WAN for internet connectivity. For portability and comfortability, Smartphone or PDA, or handheld PC that has GPRS facility is deployed as a gateway for monitoring patient records via wearable medical gadgets ${ }^{[17] .}$ Furthermore, it seeks ZigBee or Bluetooth infrastructure for communications. The network coordinator manages WWBAN including handling body wearable sensor settings including node registration, initialization in allocating sampling rate and, customization such as managing userspecific calibration, user-specific signal dispensation upload with secured data transmission via secured key exchange protocol. Interestingly, ensuring nonstop monitoring of end users' health condition, and provide instantaneous real-time feedback. Meanwhile, broad-band internet connectivity is required for static residential gateway or PC. When internet link is unavailable, PS still process and identify unusual changes, and alert patient of imminent life frightening physiological state.

\subsubsection{Trend in Health Monitoring Systems}

The following are some trends in wearable health monitoring systems; remote therapeutic services, emergency response systems (ERS), home rehabilitation and infection monitoring, video diagnostic consultation as illustrated by Frost \& Sullivan in their work.

\subsubsection{Information Rate}

Wireless setup is used for event-based supervision, where things happen at unequal 
occurrences. In comparison, wearable health tele-monitoring are positioned for recording human's essential biological signals in sporadic occurrences.

\subsubsection{Latency}

This condition is done by the applications, and sometimes traded off to enhance reliability and energy utilization. However, power conservation is crucial, replacing batteries is easily done than for wireless network, whose sensors are really hard to implement after establishment. It is important to minimize battery use to the detriment of higher latency rate.

\subsubsection{Portability}

Users have unlimited movement while under medication in wearable healthcare monitoring platform. Unlike wireless network, sensor hubs are typically viewed as motionless.

\subsection{Health Monitoring Systems Challenges}

\subsubsection{Sensitivity of Sensors}

Sensor gadget is smart and sensitive to ecological stimulations including emergency situations (fire outbreak, earthquake, nuclear factories). Some ecological settings are hash to biomedical sensor gadgets affecting patients' continuous inspection. Medical sensors must be adaptable and sensitive to ecological factors. For instance, nuclear reactor disaster, and volcanic eruption, chemical impedes transducers of sensor gadgets greatly, resulting to poor result and recalibration. Therefore, continuous effective repair mechanism enhances instantaneous well-being monitoring of crucial patients' body as pervasive healthcare platforms.

\subsubsection{Vitality of Batteries}

Efficient health monitoring demands vigorous and reliable low-energy system is serious concern for modern healthcare domain. Sensors are to be worn; bulky size hinders portability, and mobility for user. Considering battery's capability is relative to its size, even though several works done in designing low-energy sensors platforms. It is high time to start designing sensors that utilize body heat for cost-effective pervasive healthcare platforms in replacing conventional sensor battery. 


\subsubsection{Data Collection}

The massive accumulation of patients' data requires proficient data compression before transmission. Real time effective data accumulation and superb data compressibility for patient records are vital aspect in medicare. Meanwhile, methods like time-stamp of events, synchronization, packet loss, and security are open research areas for sensor development.

\subsubsection{Bandwidth}

WWBANs normally requires low-data transmission due to gadgets associated with it usually have low-memory capacity. E.g., our phone, or PDA acts as gateway or network coordinator. If the massive data gathered are not effectively compressed and remove unnecessary sub-bands before transmission, bandwidth issue will result and subsequently slow down transmission rate. Sensor nodes functioning at $250 \mathrm{Kbps}$, reducing energy utilization will result to duty cycle mechanisms. Massive data result to bandwidth issue as evident in transmission of well-being diagnostic imaging samples requiring up to Mbit/s. Therefore, an improved or efficient data compression framework implementation for multimedia data communication is needed.

\subsection{Autonomous Challenges for health Monitoring Systems}

Autonomous challenges are challenges that are not specific to any particular layers, but cut across system. Some remedies and recommendations are highlighted in subsequent subsections.

\subsubsection{Security}

Securing patient records, data compressibility and transmission in WWBANs are crucial challenges for healthcare applications especially the protection of medical information from an adversary or unauthorized user. Latest IEEE 802.15.16 standard in WBANs attempts in solving security issues but has several drawbacks. One proficient approach is security in health monitoring platform is by encrypting compressed patient data before and after transmission using Data Encryption Standard (DES), Advance Data Encryption Standard (ADES), Elliptic Curve Cryptography (ECC), and RSA to enhance security. Meanwhile, data confidentiality, data integrity, data accountability, data availability and access control are the essential security prerequisites in attaining 
our report goal.

\subsubsection{Data Confidentiality}

Securing patient related data revelation from unapproved party and entity requires data confidentiality. The likelihood of eavesdropping sensitive records conveyed via network should be stopped to secure patient health records. Deploying hash function, and digital signature to encrypt information over protected channel renders data confidentiality.

\subsubsection{Data Authentication}

Medicare and non-medicare applications require data verification. The body device needs to affirm whether the information is from genuine sensor, or from an adversary. Symmetric scheme achieves information verification.

\subsubsection{Data Integrity}

Medical records gathered over wide range should be exact, complete and consistent. Data worthiness is of awesome significance on the grounds that it is utilized to recognize and track patients as they move from one level of care to the next. Data approves personality of user to ensure patient is on medication, and to maintain billing activity. Data trustworthiness (integrity) is accomplished through data affirmation conventions, guaranteeing the received information not modified by adversary before, during or after transmissions. Digital signature hinders adversary of replaying previous messages ensuring data trustworthiness.

\subsubsection{Privacy}

Data protection for WWBANs is the procedure whereby unauthorized users are denied access, and determines whether patient related data are to be shared with third parties. It utilizes non-cryptographic scheme to guarantee security in WWBANs. Moreover, in scenario where patient health related images are processed on-nodes and only the required image is transmitted to medical coordinator improves comfort, and privacy for patients' records.

The state-of-the-art medical facilities hinder mobility because of wired and bulky medical gadgets. Health tele-monitoring framework grants maximum mobility without 
restricting movement.

\subsubsection{Internet of things (IoT)}

Tele-monitoring platform operates as result of IoT. International Telecommunication Union (ITU) ${ }^{[18]}$ referred to IoT as an era of information which links people and things, and/or exists among things, that "links world's objects in both a sensory and an intelligent manner." IoT essentially contains three layers: application, network and sensor layers respectively [19], [20], deployed into various healthcare applications. Sensor layer constitutes of actuators that assemble patient body samples and relay them to $\mathrm{CNC}$ for computational analysis. The network layer lays in-between sensor and application layers, accelerates communication of several sensor hubs to medical centers/servers for additional analysis. The application layer handles managerial tasks such as body samples recovery, storage, aggregating, and transmission of finished data to medical experts/users.

Few applicational areas are medical domains, disaster situations, multimedia applications, transportations, military surveillance, architectural designs, and agricultural appliances etc. LinkSmart is a free middleware, initially created by EU Hydra project, allows interfacing heterogeneous gadgets to consistently interoperate with extra layers of IoT in view of web domains. This thesis explores IoT in achieving efficient and portable wearable health tele-monitoring platform offering affordability with little but effective power consumption.

\subsection{Thesis Rationale}

Health tele-monitoring of fundamental well-being indicators are crucial in solving numerous drawbacks (gadget cost, lightweight medical sensors) in health platform. The demand of man's continuous misuse of universe's resources in exploration of knowledge has created devastating consequence (climate change) causing various chronicle illnesses. Meanwhile, the rocketry healthcare expenditure and incremental growth in human population have given governments globally taking proactive stance to render its citizens with inexpensive, and efficient healthcare platform that constantly monitor people's health status at anyplace, and anytime via health tele-monitoring system. Redesigning existing medical applications that counter sicknesses in a readily proactive manner are pivotal for nonstop patient monitoring that is effective and affordable. The 
huge patient data amassed are compressed via DWT and Haar in Matlab.

\subsection{Outline of Research Structure}

The remaining chapters of the thesis are: Chapter 2 explains wavelet model and literature. Discusses detailed knowledge revealing the focal concept of our research. Lossyless compression is applied in attaining efficient health monitoring. Chapter 3 describes the methodology of the research. Chapter 4 discusses the implementation and testing in Matlab platform. Chapter 5 illustrates the conclusions, recommendations and suggestions.

\subsection{Conclusion}

Modern image compression frameworks such as lossyless compression (DWT) and Fourier Transform has efficient superiority in regaining original signal image efficiently. DWT decreases storage space facilitating several images to be store disk, and also reduces the transmission interval, reduce medical gadget size and reduce hospitalization cost with comfortability mobility for users. 


\section{Chapter 2 Wavelet Model}

\subsection{Introduction}

This chapter talks about wavelet analytics approach. The research report elucidates different types of wavelets and also throws light on medicare image compression with respect to merits and demerits data compression.

\subsection{Wavelet Model}

Most medical image signals portray attributes the time space domain with no information about the frequency. This poses difficulty for both time and frequency information is needed to attain optimal compression result. Wavelet analysis split image signal into approximation and detail information. Approximation portion indicates the image pixel values overview at the top left hand corner with the other three details(horizontal, vertical and diagonal) showing the changes the medicare image undergo from one stage to another. Normally smaller detail coefficients are set to zero and exhibits negligible to the image. Thresholding assign values below detail which is said to be small to zero. Many zeros or sparsity in the medicare image provides superior compression. Power compaction is number of records achieved for both compression and reconstruction process equivalent to sum of the square pixel values. Noting $100 \%$ power preservation is Lossless which maintain image quality without depreciation and Lossy compression if the energy retention greatly reduced as in TV. A balance should be attained for huge energy loss if more zeros are to be generated.

Wavelet, a mathematical utility which reduces and partition data into many subband frequencies. This method is necessary as biological records are investigated on diverse scale or resolutions called mutiresolution, and allows mutiresolution decomposition. DWT has predominantly dominated medicare image compression field lately due to its recovering capability for image compression successfully with many mother wavelet representations. DWT coefficients are almost put to zero fluctuating up and down along the $\mathrm{x}$-axis, and contain compact signal formation ensuring our image is not over obtainable with many equals zero making the algorithm more reliable for better recovering. 
In wavelet transform, detail frequency of image signal is achievable at any particular interval and in Fourier transform, only the amplitude signal is noted while time frequency is discarded ${ }^{[21]}$. Features like matching pursuit method, basis pursuit, $\ell_{1}$ optimization method, mean square error, and normalized mean square error play fundamental function in data reconstruction scheme. Each representation present different feature and therefore, programmer need to select appropriate scheme for particular task. Wavelet function $\Psi(t)$ contains two main features ${ }^{[8]}$;

$$
\int_{-\infty}^{0} \Psi(t) d t=0
$$

For oscillatory function or wavy situation.

$$
\int_{-\infty}^{0}|\Psi(t)|^{2} d t<\infty
$$

Majority of energy in $\Psi(t)$ is partial to finite periodicity.

The speedily technological development in compression of big data integration ${ }^{[21]}$ creates an enhanced optimization scheme to attain superior data reconstruction routine. WT splits medical records into block wavelets and discards unnecessary information, maintaining relevant details for recovering. Many wavelets exist but our focus is DWT though other such as Haar, Fourier transform and Fast Fourier transform were considered to choose the scheme. Compressing image is essential because, most images contain artifacts demanding huge storage capacity and high financial cost. Compression helps eliminate redundancy, reduce transmission rate, and subsequently lesser storage space to accommodate many image signals.

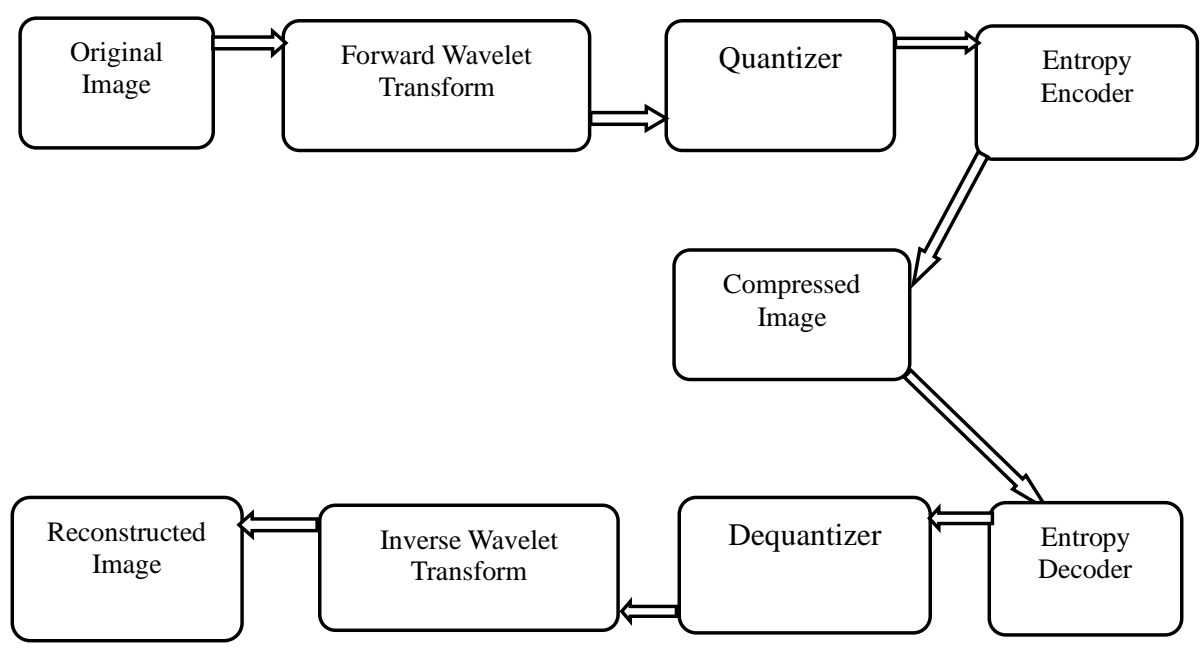

Figure 2-1 Wavelet Image Compression Framework. 


\subsection{Types of Wavelet}

Several researches are carried out lately in the different wavelet types as to determine which one is dominant over the other multiple performance indexes like compression ratio etc. Depending on which filed of compression interest you, but medicare image compression requires effective data compression and decompression technique whilst maintaining the file quality. To employ but to name few are;

\subsubsection{D Wavelet Analytics}

Medicare images constituent of 2-Dimensional samples with horizontal and vertical view alteration, and is extensively applicable in healthcare imageries. It utilizes wavelet in detail phases for all levels in the decomposition. 1D image removes high frequency from low frequency data for each decomposition level resulting to two image records. Likewise, 2D images are a matrix with $\mathrm{N}$ and $\mathrm{M}$ dimension representing rows and columns respectively. Horizontal image decomposition is filtered at each level and the details and approximation coefficients filtered on the column.

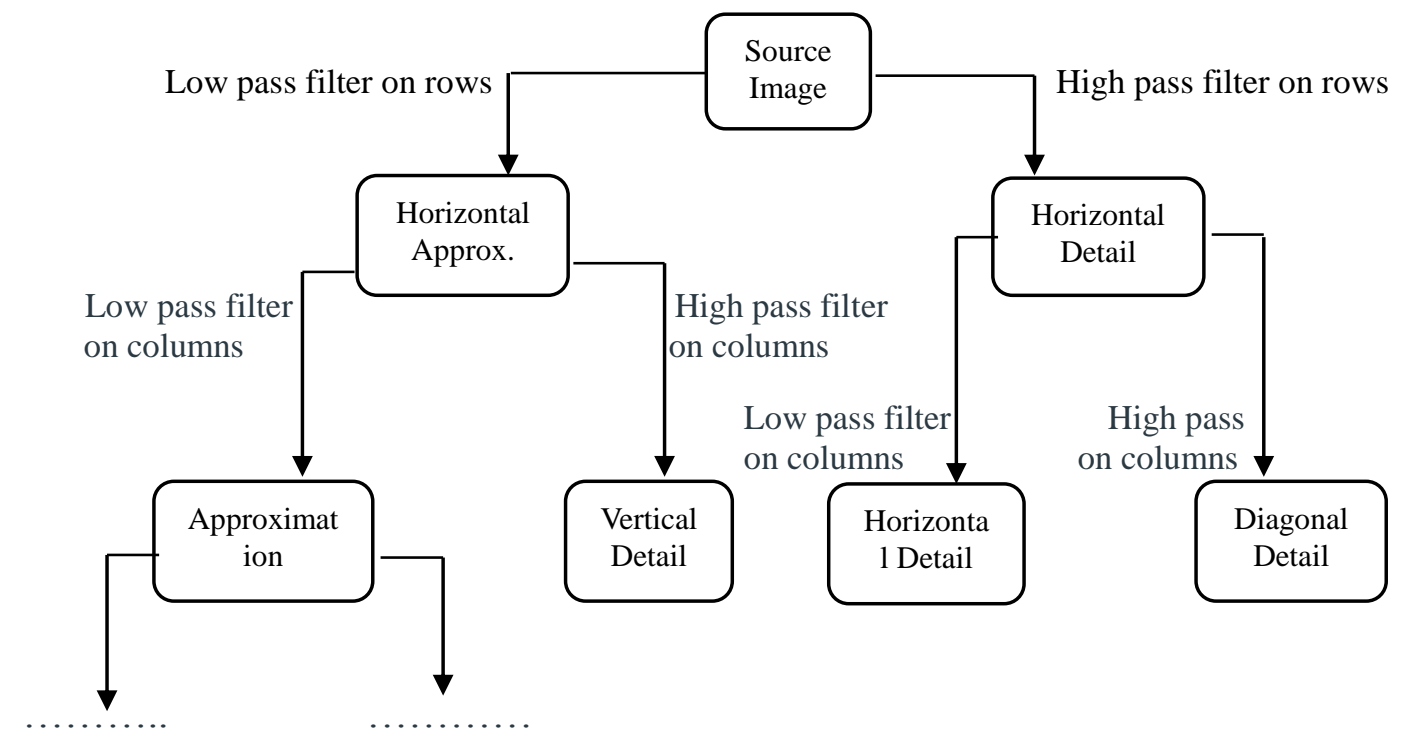

Figure 2-2 Approximation and Detail Framework

Four sub-band images are generated for each level, i.e.; Approximation, Vertical Detail, Horizontal Detail and Diagonal Detail. A picture of resolution 145 x 142 with size 44.5 KB named 100100100 of PNG format was decomposed to form the four subsignal images. This can be achieved using the wavemenu toolbox kit. 


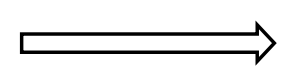

Figure 2-3 DWT Decomposition - level 1

\subsubsection{Fourier Transform}

Fourier Transform computes time frame to frequency fields allowing frequencies visibility. It is seen like a scientific prism disintegrating signal time into frequencies. Unfortunately, it lacks sufficient information about the frequencies which are interpreted unconventionally ${ }^{[22],[23]}$.

$$
\hat{f}(\xi)=\int_{-\infty}^{\infty} f(x) e^{-2 \pi i x \xi} d x
$$

For any real number $\xi$.

$$
f(x)=\int_{-\infty}^{\infty} \hat{f}(\xi) e^{2 \pi i \xi x} d \xi
$$

For any real number $\mathrm{x}$.

$\mathrm{x}=$ time,$\xi=$ frequency, $\mathrm{f}=\mathrm{FT}$ and $\hat{f}$ is the inverse transform

It is not applicable for non- stationary signals as their frequencies are not easily seen in time space, as they only indicate the frequencies without its occurrence time. It is inadequate for stationary signals as frequencies constituents arise always, and instrumental for FT image signal compression. Note, Fourier Transform exhibits poor time resolution and contains good frequency resolution. Images are split into sine and cosine wavelet with distinct frequency information successively rewriting image signal 
to different sine and cosine waves. A wavelet analysis undergoes similar process with a mother wavelet where image signal is transformed to shift and scale types of the mother wavelet.

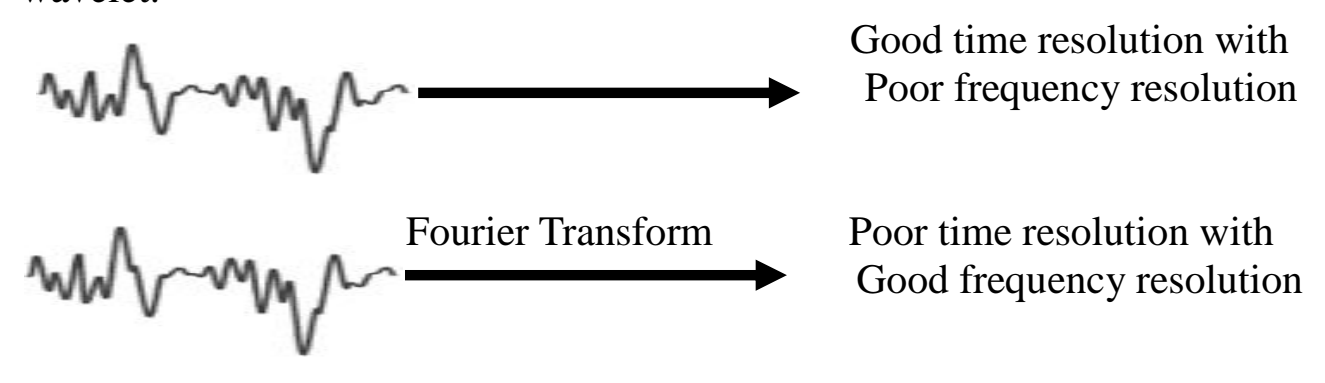

Areas of low frequency resolved well in the frequency domain but

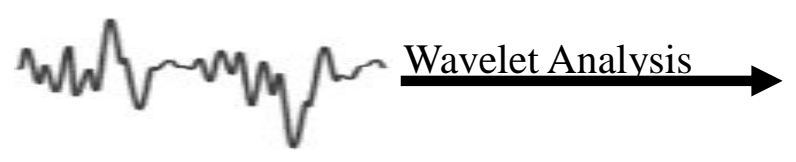
have poor time resolution

Areas of high frequency have good time resolution but have poor frequency resolution.

Figure 2-4 Fourier Different Phases

\subsubsection{Fast Fourier Transform (FFT)}

FFT is a scientific tool that transform image in time frame to frequency space. A source signal $\mathrm{f}$ of $\mathrm{N}$ variables, the computation for $\mathrm{N}$ discrete Fourier addition requires $\mathrm{N}^{2}$ complex multiplications and additions.

$$
\hat{f}[k]=\sum_{n=0}^{N-1} f[n] \exp \left(\frac{-i 2 \pi k n}{N}\right), \quad \text { for } 0 \leq k<N \quad 2-5
$$

FFT lessens statistical difficulty to $O\left(\operatorname{Nlog}_{2} N\right)$.

\subsubsection{Power Spectral Density (PSD) Estimation}

PSD estimation converts power time signal into frequency detail signals. It uses FFT where quantifiable time samples of a signal are present with higher signal to noise ratio. Figure 2-5 shows graphical illustration 


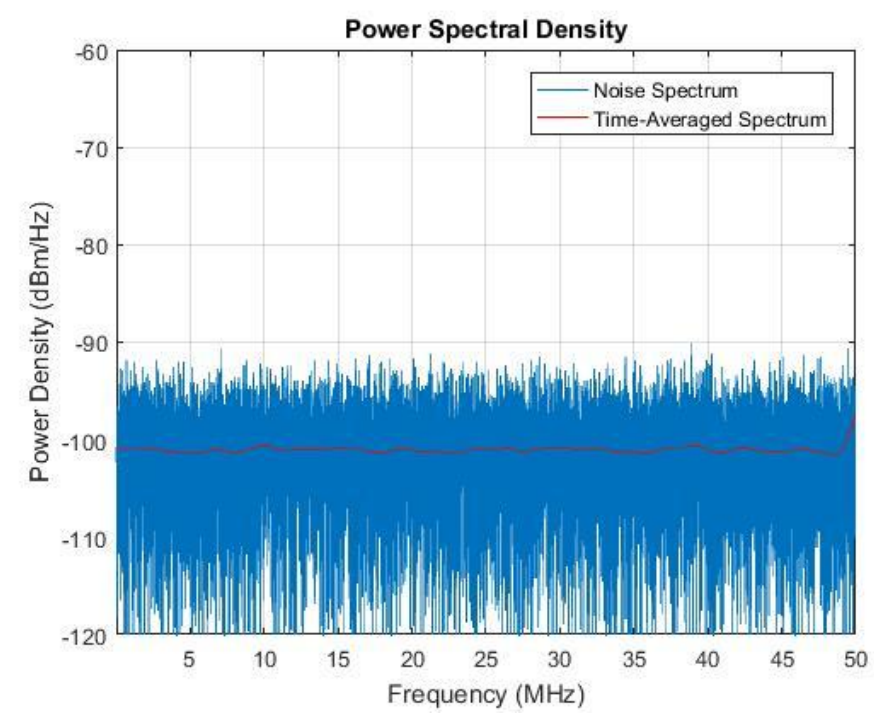

Figure 2-5 Power Spectral Density

\subsubsection{Discrete Wavelet Transform (DWT)}

DWT has better compression ratio and used in most medical and security applications. DWT is a novel phenomenon with several mother wavelet functions.

DWT is afterward used to eliminate duplication copies in image for final compression. Compressing image requires the programmer selecting the right mother wavelet functions. Compressing high excellence image means you have more details about the sample at hand. Discrete wavelets analytics apply filter banks. The sample resolution is tailored using filtering, and scale altered using up-sampling and downsampling method. High-pass filter maintains high frequency, discarding low frequency records. Low-pass filter retains small frequency data and eliminate high frequency data. The record is further stale in two sub-bands (high and low frequency). The sub-signal obtained from low filter has maximum frequency half the source image signal. This mean half source image signal is needed to regain the source image. This reduces the resolution time. The estimated sub-signal is placed in the filter bank repetitively until the desire intensity of decomposition is attained. We finally get our estimated coefficients summing sub-signals and knowledgeable sub-signals together, and the source image obtained. 


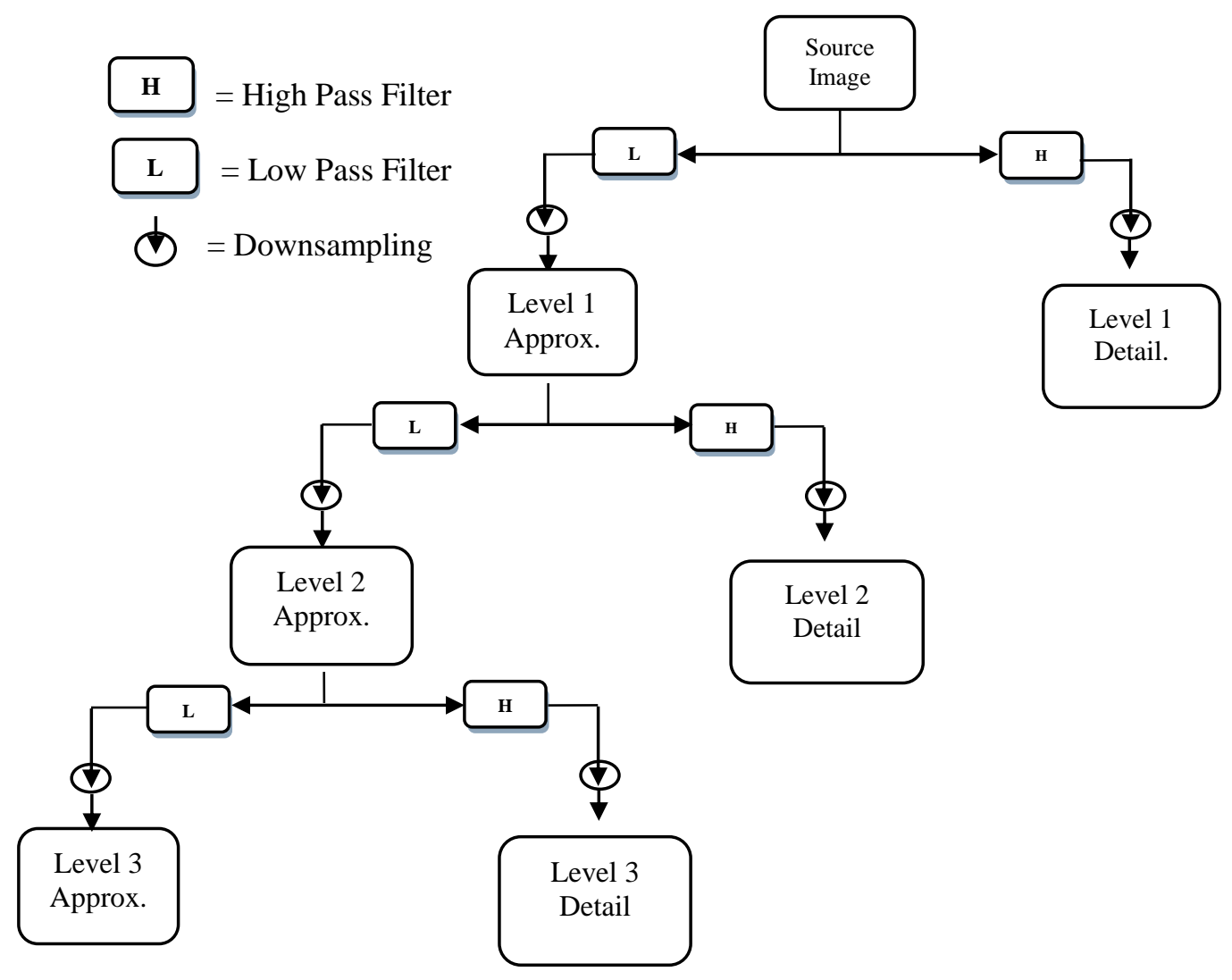

Figure 2-6 Wavelet Detail and Coefficients Sub-signal framework

\subsubsection{Discrete Wavelet Series}

First our images are discretized before compression for high superiority performance. Using Nyquist Sampling Theory ensures no signal is lost throughout the compression. High pass samples get lesser sampling percentage. The new sample rate is achieved using;

$$
N_{2}=\frac{S_{1}}{S_{2}} N_{1}
$$

$S_{1}$ and $S_{2}$ are the initial and final scales respectively, implying each scale has different sampling rate. The series are then calculated after sampling the image. DWT is afterward used to eliminate multiple duplication in the image for final compression.

\subsubsection{Merits of DW Analysis}

$\checkmark \quad$ Provides more storage space

$\checkmark \quad$ Increase transmission of patient data

$\checkmark \quad$ Easy to compress and decompress files

$\checkmark \quad$ Ease the mathematical difficulty 


\subsubsection{Demerits of Discrete Wavelet Analysis}

$\checkmark$ Sometimes results in transmission error

$\checkmark$ Always require to decompress the body signal before making judgment

$\checkmark$ Sometimes results to complication

\subsubsection{Limitation of Discrete Wavelet Analysis}

Though DWT is a power tool used in data compression as evident by the FBI of the United States used to compress fingerprint records, yet still, it has few limitations such as;

\section{$\checkmark$ Shift Sensitivity}

It affects DWT from the down sampler (any shift in time for physiological records alarm unpredictability and make the DWT coefficients to fail in analyzing between physiological record shifts.

\section{$\checkmark$ Poor Directionality}

DWT attributes poor directional selectivity for diagonal features.

\section{$\checkmark$ Phase Data}

Normally affect phase data especially in image compression which is calculated in real and imaginary projections.

\subsubsection{Haar Wavelet}

Haar wavelet is a simple wavelet from mother wavelet with discontinuous function, and possesses identical wavelet like Daubechies db1. It decomposes image into two coefficient components, approximation (averaging) and detail (differencing). It works as an input size of $2^{\mathrm{n}}$

Haar exhibits a unique structure in Equation (2-7)

$$
\psi(t)=\left\{\begin{array}{c}
1\left(0 \leq t<\frac{1}{2}\right) \\
-1\left(\frac{1}{2} \leq t<1\right) \\
0 \text { (otherwise })
\end{array}\right.
$$

2 Phases for processing;

Separate horizontally

Separate vertically 


\subsubsection{Mutiresolution}

Nevertheless, Mutiresolution scrutinizes full signals in same sphere of different resolutions. Higher frequency constituents signal utilize small window in attaining optimum time resolution, and small frequency constituents use larger window in achieving superior frequency details. Vitally, the window has identical capacity regardless of height and width with different wavelet analytics. Heisenberg's Uncertainty Principle controls the window's area especially when the frequency resolution increases and correspondingly decreases the time resolution. Mutiresolution a fascinating pillar for wavelet analytics.

\subsubsection{Energy Consumption}

Energy Consumption Wavelet transform energy compaction is optimum. Image energy is summation for all the squares pixel values in image. For wavelet transform is a summation of transform coefficients in an image. Energy is partitioned into approximation and detail samples without alteration in total energy. However, some energy are lost in compression i.e.; threshold changes coefficient values resulting to minimal energy lost in compressed image. Energy compaction in detail coefficients drastically lower than approximation coefficients.

\subsection{Data Compression}

Data Compression is a procedure involve minimizing data to reduced bits of data pixel points within the data to be stored for further analysis.

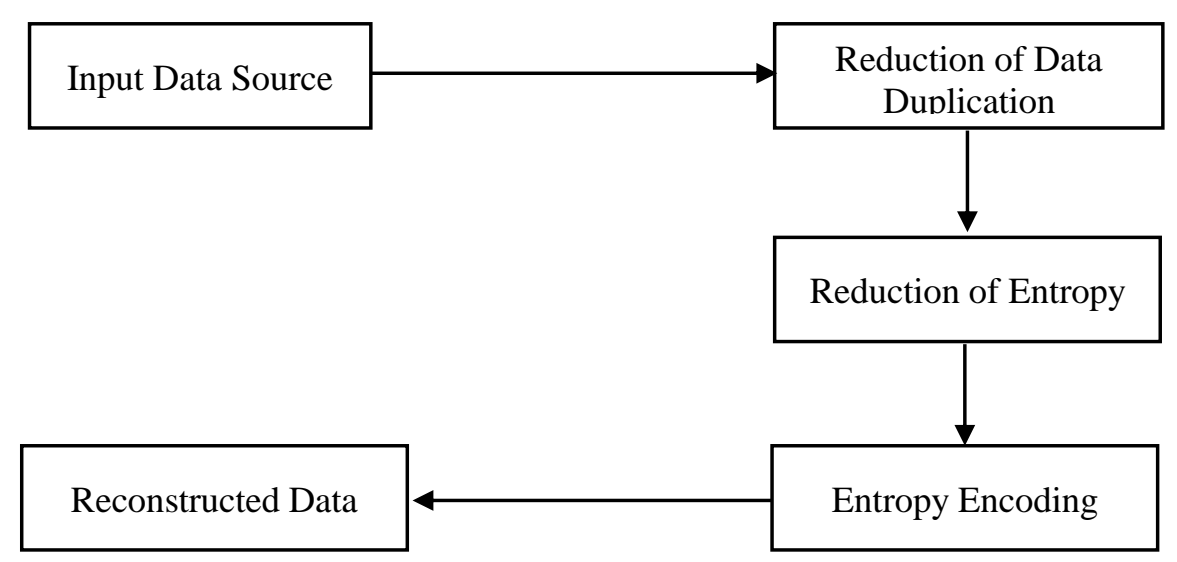

Figure 2-7 Data Compression Structural Model 


\subsubsection{Data Compression Advantages}

Data Compression play important role for health monitoring systems. The numerous challenges and setbacks in medicare image big data are achievable with the appropriate data compression technique. Some advantages are in;

1. Remove duplicate pixel points within data set.

2. Minimize medicare storage requirements

3. Lower of operation in terms of data processing.

4. Limit the data transmission interval on the networks.

5. Increases the quality and efficiency of data.

6. Facilitate of the use of video-conferencing, e-consultancy, health monitoring applications, and used in many multimedia applications.

7. Easy of querying a particular data.

8. Reduces hardware design implementation.

9. Ease the mathematical computational difficulty.

\subsubsection{Data Compression Disadvantages}

Though data compression has proven to be an efficient tool for medicare application, but there still exist some constrains such as;

1. Encoding and decoding problems in some region.

2. Interpreting the compressed data

3. Always perform decompression for of data.

4. Data transmission error result to misinformation.

5. Security problems.

6. Addition of noise to processed data.

\subsection{Conclusion}

Traditional data compressibility yields little effects on health monitoring system. Other constrains like bulky wired devices attached to patients restrict mobility and comfort, poor image quality, and inability to constantly monitor patient out of hospitals. This report shows a highly efficient image compression can be achievable when DWTapproximation information is utilized well. Compression-based algorithm skillfully 
recovers signals \& imageries with little alertness of the measurements' sparsity with excellent quality.

Nevertheless, there exist few drawbacks in healthcare image processing for remote health tele-monitoring systems. Non-sparsity of few samples recorded in remote health tele-monitoring pose major challenge. 


\section{Chapter 3 Methodology}

\subsection{Introduction}

The proposed implementation uses DWT analytics. The focus is DWT and Haar wavelets for our proposed implementation.

\subsection{Medical Image Compression Overview}

The widely used applications of images in medicare fields ranging from MRI, CTs, medical tele-conferencing, video-conferencing and e-therapist consultancy raises certain questions which need answers. Huge healthcare records such as images, graphics, and

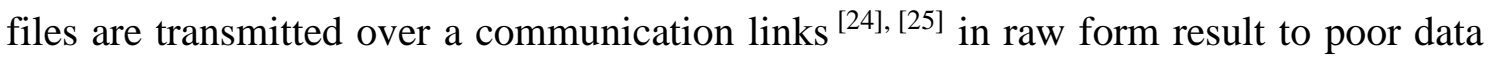
transmission interval, increase in storage space on disk and increase in cost of operation. Medicare imageries constituents of many artifacts called redundancy or duplication which consume more of storage space on disks, increases transmission bandwidth and require more time for both uploading and downloading processes.

Therefore image compression answers those questions. Medicare image compression effectively compresses and decompresses images in medical fields. Medicare image compression can either be lossy or lossless compressions which are further explain in preceding subsections. Lossless compression includes Huffman coding, LZW coding, Entropy coding, Bit-plane coding and Run-length coding and lossy examples are fractal compression, wavelet transform, transform coding, Fourierrelated transform and discrete cosine transform.

\subsection{Current Methods}

Due to time factor, only limited current systems will be dealt with and we will focus more on our proposed implementation scheme.

\subsubsection{Discrete Cosine Transform (DCT) Based Coding}

DCT offers approximation reveals Discrete Fourier Transform (DFT) real portion of the series. For instance, in a given set of data of N, the DCT's computational periodicity is of order $N \log _{2} N$ resemble DFT but with optimal convergence than DFT. 
Figure 3.1 shows an image initially decompose into an 8 × 8 partitions allowing forward discrete cosine transform (FDCT) performed on each block partition. FDCT concentrates on image signal in lower spatial frequencies whose number of bits are zero or close zero. The coefficients are then finally quantized and encoded to attain the compressed image, and the revise produces of IDCT achieved the decompression process.

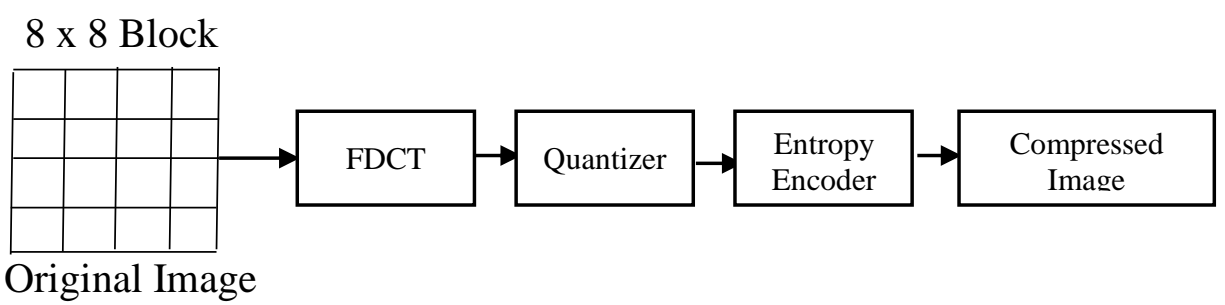

Figure 3-1 DCT Image Compression Architecture

\subsubsection{Discrete Wavelet Transform (DWT) Based Coding}

Wavelets ${ }^{[26],[27],[28]}$ represent medicare image into detail and average coefficients. Compressing an image rests on the wavelet type feature in implementing a task. The number of data pixel point in wavelet is considered to be $N=2^{n}$ with $\mathrm{n}$ being the positive integer. Haar wavelet exhibits nearest neighbour in level-1 high-pass and lowpass coefficients which can be written to level-2 and so on.

\subsubsection{Huffman Based Coding and Decoding}

Huffman coding uses a frequency binary tree system which is an optimum compression scheme ${ }^{[29]}$. Huffman coding exploits two least symbols in the alphabet and combine them to unitary symbol and repeat the processes until final result is obtained. It is a process for coding symbols based on their numerical event (probabilities) with each pixel as a symbol. Huffman coding is a prefix coding processing assigning most frequency symbol with smaller bits and least frequency with bigger bits. The inverse process is the Huffman coding which decompose and reconstruct the source image. Huffman code is done using the following steps; Rank all symbols in descending order of probabilistic occurrence, effectively add the two symbols of least probability to form the new symbol (source reduction) and build binary tree with each node as the probability for all the nodes, and finally track the root for 
each leaf in the direction of their nodes.

\subsection{Proposed Compression Method Implementation with (DWT)}

Medicare image compression is fundamental for establishing health monitoring applications for communication with compact data size. Therefore, our report is rooted on wavelet applications. The sample image is initially decomposed into sub-band coefficients ${ }^{[30]}$. The sub-band coding, input frequency are split into band limited compartments and differentiate low-pass and high-pass segments using two set of filters. The sub-band coding exploits advantage of different sensitivities of our eyes in differencing various frequency compartments. The sub-band image coefficients are comparing with the threshold, and those below the set threshold are rendered to zero, and values above the peak are encoded via lossyless compression framework.

Medicare image compression is possible in two forms; decomposing the into low and high frequency parts ignoring the high frequency part thereby recovering image again. This technique attained optimal compression ratio but with minimal distortion. The other type is Thresholding high frequency blocks and discard blocks below threshold ${ }^{31}$. The reconstructed image record exhibits superior compression ratio and eventually diminish image distortion. DWT portray efficient image compression than DCT with optimum compression ratio and PSNR and finally remove the blocking effect present in other compression techniques. Embedded Zerotree Wavelet method (EZW) is wavelet transform which encode wavelet coefficients in relation to coefficient values. Important details are first encoded follow by less important information. The bit generates embedded stream which can be terminated at any point after achieving the goal $^{32]}$, and also decoding at any stage with the image been reconstructed as the source image.

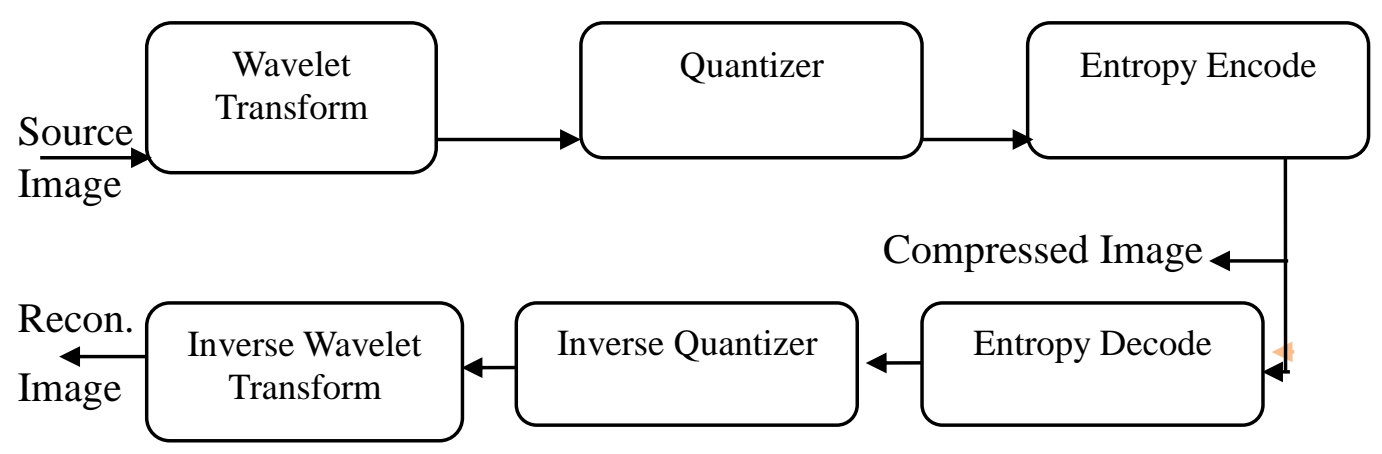

Figure 3-2 Wavelet Transform compression Framework 


\begin{tabular}{|l|}
\hline \multicolumn{1}{|c|}{ Pseudo code for DWT compression } \\
\hline A. Decomposition Stage \\
A wavelet type was chosen with N, calculate the wavelet and \\
decompose image signal at N level. \\
\hline \multicolumn{1}{|c|}{ Thresholding } \\
Hard thresholding was applied to individual level starting 1 to $\mathrm{N}$ to \\
coefficients. \\
\hline \multicolumn{1}{c}{ C. Reconstruction Stage } \\
\hline $\begin{array}{l}\text { Calculate the reconstructed wavelet from true approximation } \\
\text { coefficients. }\end{array}$ \\
\hline
\end{tabular}

\subsubsection{Digitizing Image}

Compression process starts with our image being first digitized. Digitized image is viewed by its concentration levels, or gray scale levels from black (0) to white (255) and resolution. Tradeoff is necessary in image compression to save time and finance.

\subsubsection{Thresholding}

Most wavelet coefficients tend to or equals zero. This tool modify image signal coefficient to get many zeros. Thresholding certain information in the wavelet means to discard certain detail in the image. This mean one should know how to determine the threshold and how to apply it. Two classes of thresholding in wavelet imageries compression using entropy coding.

\subsubsection{Threshold Value}

Threshold value has affected the quality for compressed image. The main concern is truncate less important detail because the quantity of data in it is negligible. Determining the threshold poses setback in the process. Donoho and co-author [33] provide the asymptotical optimal formula, the "Universal Threshold $(\mathrm{t}){ }^{[34]}$ as;

$$
t=\sigma \sqrt{(2 \ln N)}
$$


where $\sigma$ is the standard deviation of $\mathrm{N}$ wavelet coefficients. The $\mathrm{t}$ is computed for each level of decomposition for only high-pass coefficients. Low-pass coefficients are preserving to smooth additional decomposition.

\subsubsection{Applying thresholding to a value}

Two types of threshold existing in image compression are;

\section{$\checkmark$ Hard thresholding}

Coefficients below set threshold fixed to zero, and those above discarded creating more zeros. This technique reduces storage room and reduce transmission rate. It is computed as;

$$
T(t, x)= \begin{cases}0 & \text { if }|x|<t \\ x & \text { otherwise }\end{cases}
$$

Values for $\mathrm{x}$ less than threshold $(\mathrm{t})$ are plug to zero.

\section{$\checkmark$ Soft Thresholding}

In soft thresholding, all image signal coefficients $x$ lesser than threshold $(t)$ are put to zero and subtract $\mathrm{t}$ for all $\geq t$. It is seen as transformation of signal approaching zero through tolerance h. A mathematical depiction is;

$$
T(t, x)=\left\{\begin{array}{cc}
0 & \text { if }|x|<t \\
\operatorname{sign}(x)(|x|-t) & \text { otherwise }
\end{array}\right.
$$

Soft threshold produces optimal PSNR than hard threshold.

\subsubsection{Entropy Coding}

No compression is done in wavelet and thresholding stage. Wavelet analytics only give detail information concerning the targeted image signal to be processed, confirming the "compressed sensing theory" which states that giving little details about signal, the replica image signal can be reproduced. Huffman coding, a standardize skill to process image, because its data points are small and essentially zero. Large numbers are assigned with additional bits and smaller numbers allocated with lesser bits. More zeros result to efficient compression ratio.

\subsubsection{Quantization}

Quantization a technique where an image signal's values are digitized. This technique converts string fluctuating points $\mathrm{w}^{\prime}$ toward series for integers $\mathrm{q}$, smallest 
ones are rounded-up to the adjacent integers. It produces higher compression ratio and a one-to-one function $Q(x)$ mapping several input information into smaller output values. Quantizer set numbers $\left\{d_{i}, i=0, \ldots, N\right\}$ labeled as decision points and set of numbers $\left\{r_{i}, i=0, \ldots, N-1\right\}$ called reconstruction levels. Input value $\mathrm{x}$ is mapped to a reconstructed level $r_{i}$ if $\mathrm{x}$ lies in the interval $\left\{d_{i}, d_{(i+1)}\right\}$.

\subsection{Image Processing}

Image processing is a fundamental element in global information age. Majority of social apps like Facebook, Tweeter, WhatsApp, WowApps, etc. and even news channels like BBC, VOA, CCTV, SLBS etc. continuously used images every seconds in their operations. It is a process where images are processed (compressed) and transmitted through network reducing storage space, and increase the transmission speed. Image compression eliminates redundant data in an image facilitating reconstruction of image. Removing the artifact or unnecessary components before reconstruction save money, reduce storage space and speedup communication rate. Redundant detail is simply eliminating unnecessary duplications in image set for compression. Few applications are Image Compressing; Image De-noising, Image Enhancement, Image Recognition, Face Detection and Extraction, Texture Classification.

\subsection{Image Compression Problems}

Whether Lossy or Lossless compressions are faced by few constrains before attaining optimal image compression ratio. Below are few stated problems;

\subsubsection{Compression Method Used}

The implementation for health monitoring systems that relies on low network frequency such as Bluetooth or ZigBee needs efficient compression method. Choosing wrong compression method in health monitoring systems result to catastrophe consequence. Therefore, most health application uses lossless compression for its high compression ratio and data recovering paradigm. Using lossy compression produces inaccurate health records leading to wrong medical prescription.

\subsubsection{Open Standard}

This lead to a question whether healthcare monitoring systems should institute 
opens standard or compression method.

\subsubsection{Scalability}

A well-chosen compression method helps in both uploading and downloading medicare images before or after compression stages or decompression stages. The method should be scalable to meet the different system architectures to achieve reduce medicare file transmission and also diminish the storage space on disk with lesser periodicity.

\subsection{Basic Compression Types}

Essentially, medicare image compression methods are lossy and lossless compression $^{[35],[36]}$ respectively.

\subsubsection{Lossy compression}

Lossy image compression method is applied in several health applications. It encompasses numerous schemes including Transform coding, DCT and DWT. Lossy compression is not efficient as compressed image cannot be recovered source image but instead gives an approximation image. Here, sometime the programmer makes choose whether to tradeoff image data size over image quality.

$$
f(x, y) \neq \hat{f}(x, y)
$$

Lossy scheme process images to lesser size than lossless method, but attaining higher compression ratio.

\subsubsection{Lossless compression}

An improved effective compression scheme is lossless compression which processes image with super quality than lossy method. The compression ratio is low which is exactly as the original images without loss of data. The scheme exploits only the statistical duplications and maintains the image quality.

\subsection{Image Data Properties}

Comparison for countless image compression frameworks prompts researchers to know image properties. Entropy follows this category such as high correlated image 
produces low entropy. For instance, extremely squat frequency with highly correlated image optimally compressed by several frameworks. Image property is vital, and not compressibility that yields excellent compression rate. Notably, compression scheme effective for few images will not possibly be efficient for all medical images. Entropy ${ }^{[37]}$ is computed via the formula stated in (3-5);

$$
H_{e}=-\sum_{k=0}^{G-1} P(k) \log _{2}[P(k)]
$$

The data duplication $(\mathrm{r})=\mathrm{b}-\mathrm{H}_{\mathrm{e}}$

Note $b$, the least integer of bits in quantization level is achieved. And data redundancy is calculated provided optimum image entropy is present which normally do not occur frequent due to few arithmetical data is unknown.

\subsection{Image Compression and Reconstruction}

Image Compression reduces image size to reduced storability and speedup transmission rate. It has two basic structural entities; encoder and decoder. An image $f$ $(\mathrm{x}, \mathrm{y})$ enters encoder producing set of input data to epitomize image. The approximation image $\mathrm{f}^{\prime}(\mathrm{x}, \mathrm{y})$ is attained by compressing and de-compressing the image.
(a) Source Sample
(b) Compressed Sample 


\section{(C) Reconstructed Image}

Figure 3-3 Wavelet Image compression and decompression

\begin{tabular}{|l|}
\hline Algorithm: DWT Image Construction and Decomposition \\
\hline Lunch Matlab and open the wavemenu toolbox \\
\hline Load the image named brain.jpg with resolution 153 x 150 \\
\hline Choose biorthogonal wavelet type with 1.3 ratio and then select level 3. \\
\hline Click analyzes and select compress. \\
Use "By level thresholding" and select "Balance Sparsity-norm under thresholding \\
method. Also select "Horizontal detail coefficients". \\
\hline Click compress and save the image.
\end{tabular}

Table 3-1 Results obtained for figure 4-5.

\begin{tabular}{|c|c|}
\hline DWT Level & Threshold Values \\
\hline Level 1 & 250.1 \\
\hline Level 2 & 481.7 \\
\hline Level 3 & 935.3 \\
\hline
\end{tabular}


Figure 3-3, (a) is source picture, (b) is compressed sample and (c) is reconstructed image. Energy was preserved in compressing and decomposing image to $99.93 \%$ with $76.98 \%$ zeros.

Figure 3-4 three level biorthogonal sample

Figure 3-4 indicates level 1 to level 3 of our Biorthogonal medical image.

\section{Importance of Biorthogonal Wavelet}

$\checkmark$ Bior exhibits robust control on medicare image compression (compactness)

$\checkmark$ Flexibility is contained in biorthogonal wavelet analysis

$\checkmark$ Compact domain allows accurate implementation.

$\checkmark$ Wavelet and scaling function are represented by FIR filter.

Several compression performance indexes ${ }^{[8]}$ are used to simulate the effectiveness of compressed sample in Matlab in equations 3-6, 3-7 and 3-8.

$$
P S N R=10 \log _{10}\left(255^{2} / M S E\right) d B
$$

PSNR and MSE measured in Decibel.

$$
M S E=\frac{1}{M N} \sum_{x=1}^{M} \sum_{y=1}^{N}[f(x, y)-\hat{f}(x, y)]^{2}
$$

Note, MSE is mean square error,

$\mathrm{M}=$ Image rows,

$\mathrm{N}=$ Image columns. 


$$
C_{R}=\frac{n_{1}}{n_{12}}
$$

$C_{R}$ is Compression ratio,

Example, the CR 80:1 means the source image has 80- bits per unit in the compressed data.

Table 3-2 MSE, PSNR and CR values of different performance indexes for Brain

\begin{tabular}{|l|c|c|c|}
\hline Wavelet Type & MSE & PSNR & CR \\
\hline Haar & 60.25 & 30.33 & 10.9167 \\
\hline Bior & 60.25 & 30.33 & 10.9167 \\
\hline Coif & 58.89 & 30.43 & 9.7300 \\
\hline Sym & 59.03 & 30.42 & 9.6324 \\
\hline
\end{tabular}

Table 3-2 shows the comparisons among three different performance indexes using brain scan for four different wavelet types. Our result shows that coif had better MSE and PSNR than the other wavelets but sym has an optimum compression ratio.

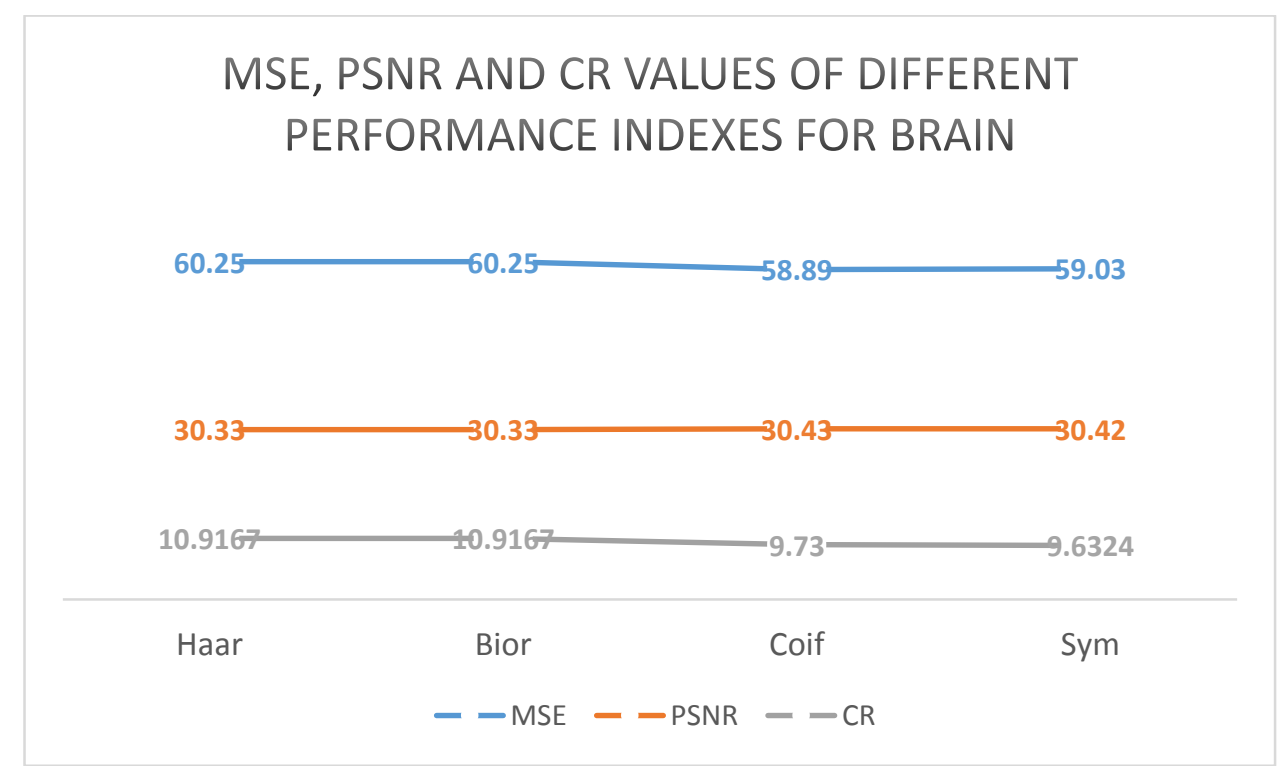

Figure 3-5 MSE, PSNR and CR values of different performance indexes for Brain 
Table 3-3 MSE, PSNR and CR values of different performance indexes for Heart

\begin{tabular}{|l|c|c|c|}
\hline Wavelet Type & MSE & PSNR & CR \\
\hline Haar & 27.78 & 33.69 & 3.7500 \\
\hline Bior & 9.97 & 38.14 & 1.2259 \\
\hline Coif & 32.45 & 33.02 & 1.8132 \\
\hline Sym & 35.23 & 32.69 & 1.8400 \\
\hline
\end{tabular}

Table 33 shows the comparisons among three different performance indexes using human heart for four different wavelet types. Our result shows that bior had better MSE, PSNR and CR than the other wavelets.

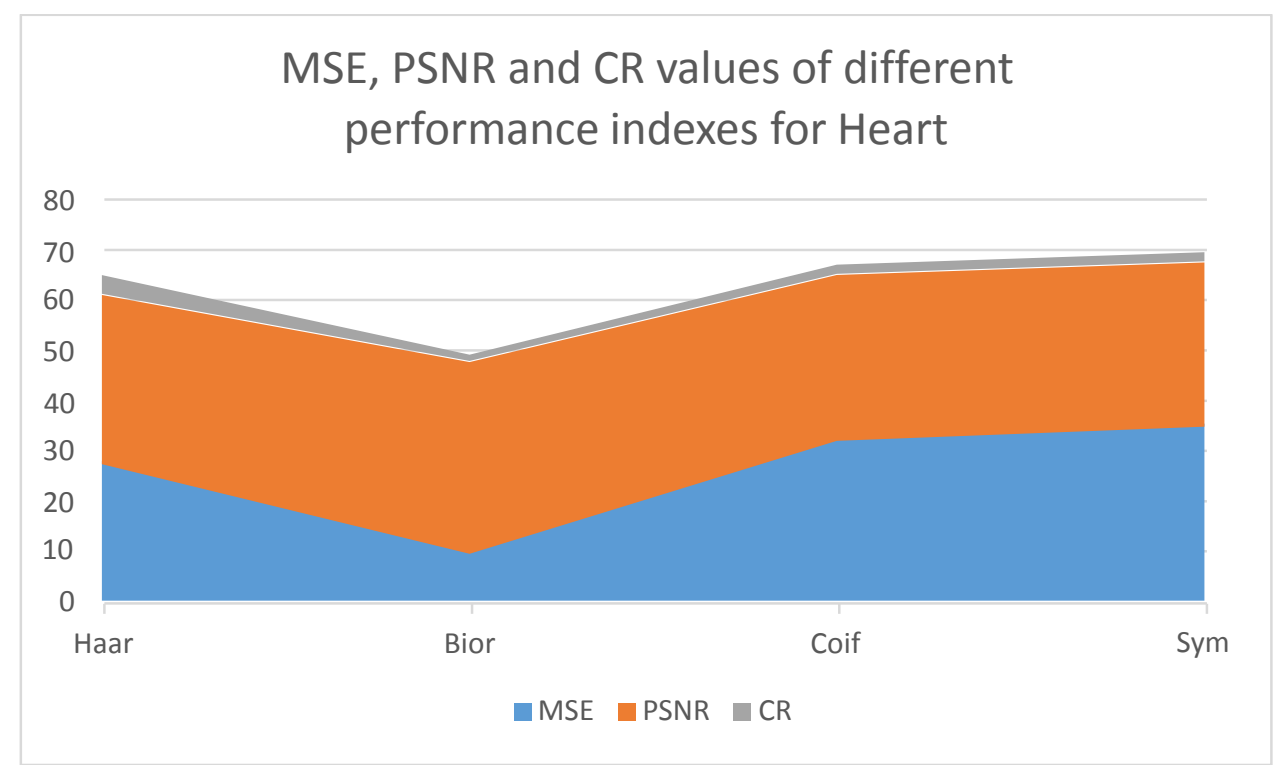

Figure 36 MSE, PSNR and CR values of different performance indexes for Heart

\subsection{Conclusion}

Our work proposed compression-based implementation called DWT for compressing medicare images, with high quality. They remove computational complexity in traditional image procession schemes. This is significant for medical imageries; i.e.; brain pulse, heart rate which needs accurate details before treatment. The exploitation of DWT with minimal power wavelet technique greatly reduced on chip computing resources relating to energy exploitation and gadget size. This research was more successful, because it was based on relevant lessons learnt in classroom such as Matrix Theory, Numerical Analysis, Stochastic Processes and Applications, Software Development and Applications, Mobile Computing rather than internet and textbook literatures. 


\section{Chapter 4 Implementation and Testing DWT-based Image Compression in Matlab}

\subsection{Introduction}

This chapter throws light on implementation and testing for DWT based compression. We proficiently attained our goal for implementing mother wavelet to compressed medicare image for transmission for health monitoring systems. The system renders its user with comfort and mobility devoid of restricting their daily activities. Our implementation method was compared to other compression schemes.

\subsection{DWT Applicability}

Nowadays, integrating extremely miniature scalable sensor, microcontroller, and radio wave included on smart fabric or synthetic have resulted to another era in health monitoring systems. These systems are used by several entities, governmental and nongovernmental institutions like Obama-care ${ }^{[38]}$, traffic control systems, agricultural research, security agencies, environmental supervision and numerous applications. Variety of body sensors supervises essential body signals, ecological sensor, and location sensor harmonized to form WWBAN in monitoring well-being conditions at distance.

\subsection{Software Implementation}

Matlab platform was used for implementing a DWT-based image compression for our research through the graphical user interface toolbox. And experimentation was successful in accurately recovery body signals with high superiority performance with loss of data image.

\subsection{Experimentation}

Different medical images were collected, store, process and analyze using different wavelet types. We focus more on DWT, Haar wavelet, biorthogonal wavelet because of limited research time. We were able to compress, decompress and de-noise medicare images using lossless compression method, because medical data need to be reliable and 
complete. Performance indexes such as CR, MSE and PSNR were applied to test the effective of our proposed DWT.

\subsection{Implementation}

\subsubsection{FFT Implementation Setup}

Step 1: Load the color image

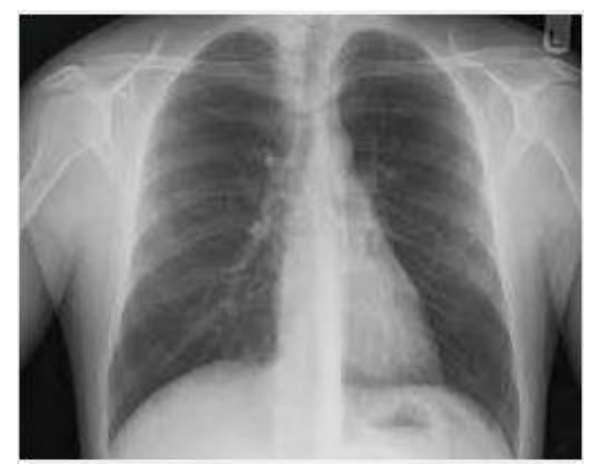

Figure 4-1 (a) Source image for our FFT

Step 2: Convert the image to black and white

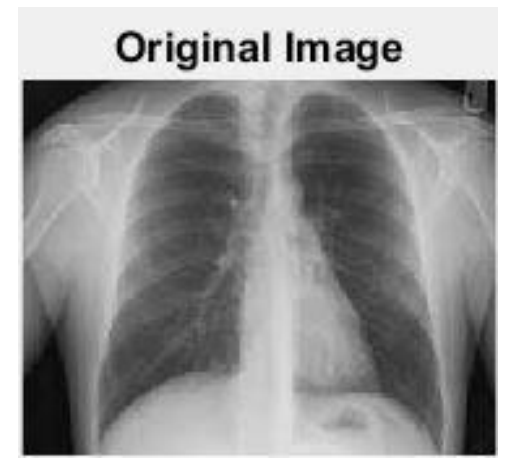

Figure 4-1 (b) Converted FFT image black and white

Step 3: Compute the FFT of our image using $\mathrm{fft}$

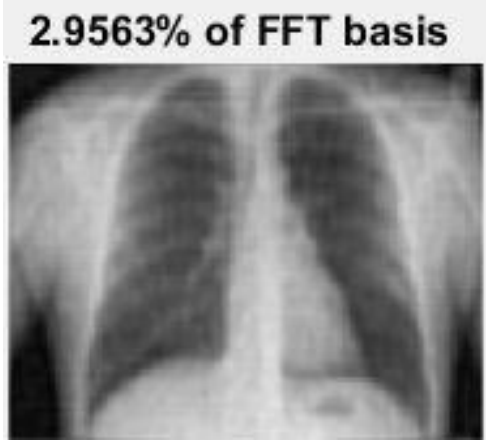


Figure 4-1 ( C) Computed FFT of our image using fft2

Step 4: Zero out all small coefficients and inverse transform

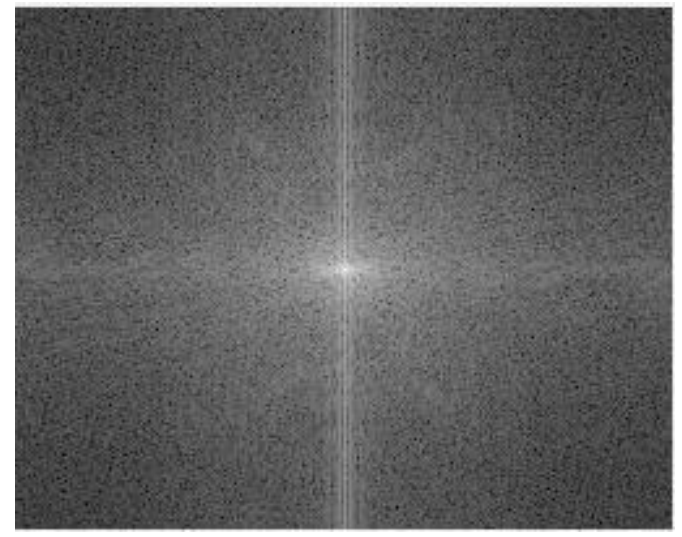

Figure 4-1 (d) Zeroing small coefficients and inverse transform

Note: Fast Fourier Transform implementation code can be found in the Appendix

\subsubsection{Energy Loss or Gain Implementation Setup}

The steps using Matlab toolbox to explain energy loss or gain are;

\begin{tabular}{|rl|}
\hline \multicolumn{2}{|c|}{ Pseudo code for the compression procession } \\
\hline$\checkmark$ & Lunch Matlab wavemenu \\
\hline$\checkmark$ & Load the image \\
\hline$\checkmark$ & Select the wavelet type sym(4) with four level \\
\hline$\checkmark$ & Set the default to "Global thresholding", select "Bal. sparsity- \\
& norm(sqrt) under thresholding and choose compress \\
\hline$\checkmark$ & Click analyze and press compress \\
\hline$\checkmark$ & Finally compress the image and save. \\
\hline
\end{tabular}
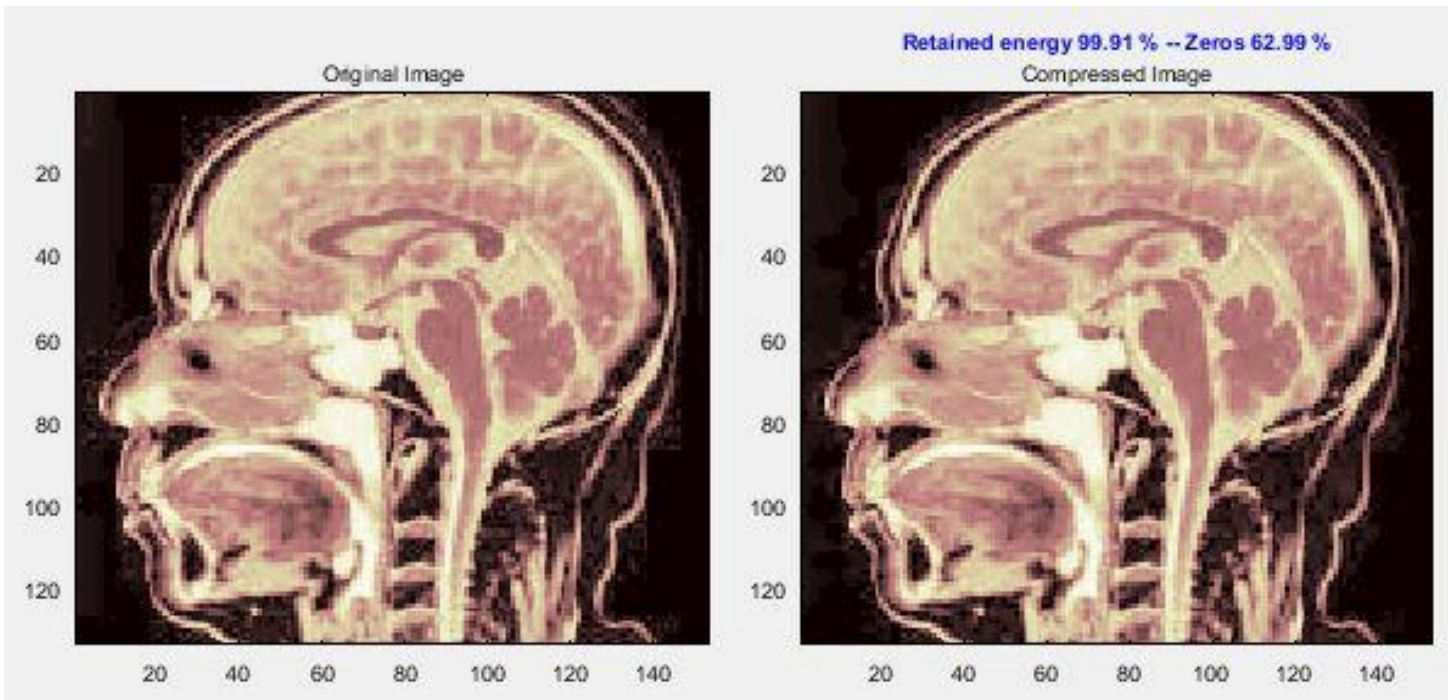
Figure 4-2 Illustration of Energy Loss or Gain

Figure 4-2 shows original image 100\% energy but after compression, some energy was lost because of compaction in approximation details to about $99.95 \%$ with $94.75 \%$ zero coefficients.

\subsubsection{Experimentation Result for Image Compression}

Our experimentation is rooted on DWT and executed using Matlab platform.
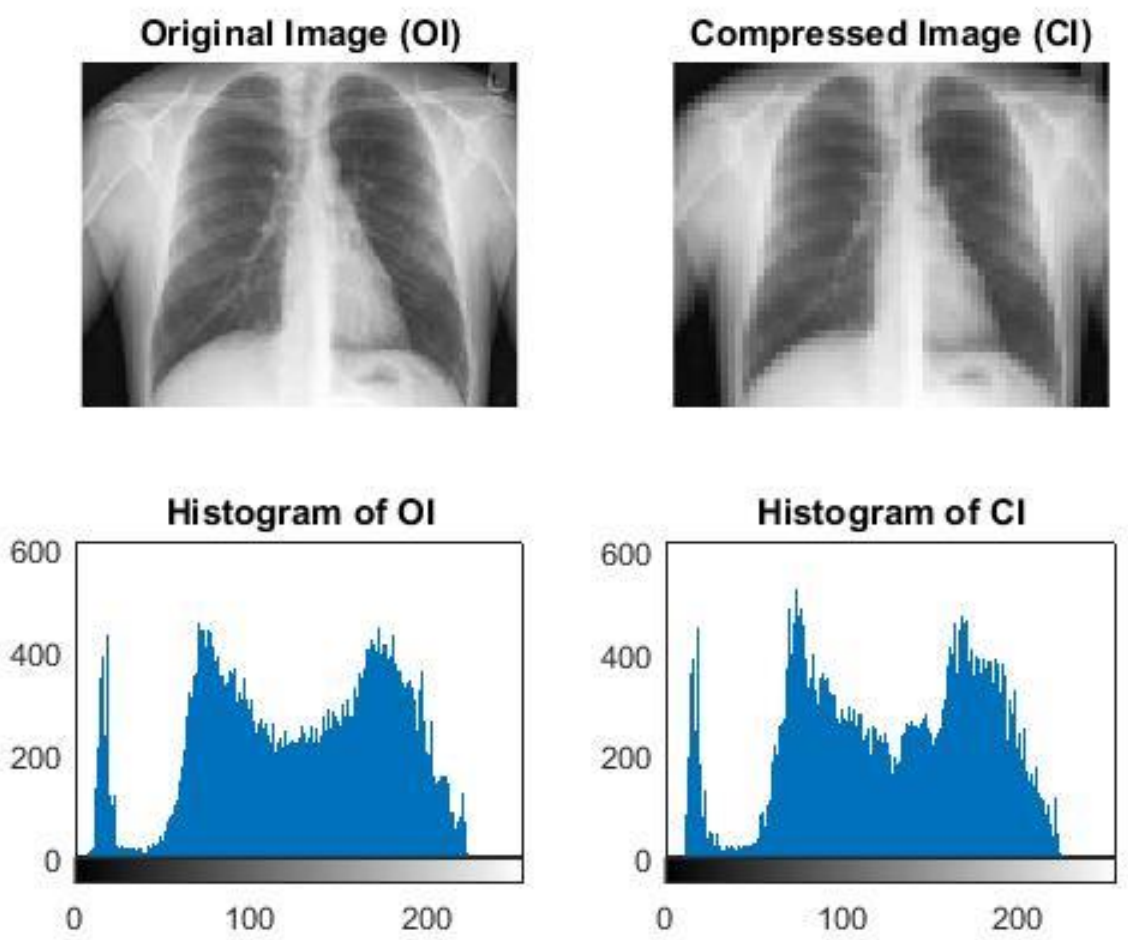

Figure 4-3 Histogram Representation

Proposed compression structure for diverse threshold standards are tabulated below showing that $\delta=50$ is an optimal point for the different compression ratio.

Table 4-1 Results obtained from different threshold standards with proposed compression scheme.

\begin{tabular}{|c|c|c|c|}
\hline $\begin{array}{c}\text { Thresholding } \\
\text { Values }\end{array}$ & $\begin{array}{c}\text { Image Original } \\
\text { Size }\end{array}$ & $\begin{array}{c}\text { Compressed Image Size with } \\
\text { DWT }\end{array}$ & $\begin{array}{c}\text { Compression } \\
\text { Ratio }\end{array}$ \\
\hline $\begin{array}{c}\text { Without } \\
\text { Threshold }\end{array}$ & $159 \mathrm{~KB}$ & $22.6 \mathrm{~KB}$ & 7.0354 \\
\hline$\delta=10$ & $159 \mathrm{~KB}$ & $20.7 \mathrm{~KB}$ & 7.6812 \\
\hline$\delta=20$ & $159 \mathrm{~KB}$ & $14.3 \mathrm{~KB}$ & 11.1189 \\
\hline$\delta=30$ & $159 \mathrm{~KB}$ & $21.1 \mathrm{~KB}$ & 7.5355 \\
\hline$\delta=40$ & $159 \mathrm{~KB}$ & $21.0 \mathrm{~KB}$ & 7.5714 \\
\hline
\end{tabular}




\begin{tabular}{|l|l|l|l|}
\hline$\delta=50$ & $159 \mathrm{~KB}$ & $13.8 \mathrm{~KB}$ & 11.5217 \\
\hline$\delta=60$ & $159 \mathrm{~KB}$ & $20.8 \mathrm{~KB}$ & 7.6442 \\
\hline
\end{tabular}

Table 4-2 Showing comparison between DWT and other compression frameworks

\begin{tabular}{|c|c|c|c|}
\hline $\begin{array}{c}\text { Compression } \\
\text { Techniques }\end{array}$ & File Sizes & $\begin{array}{c}\text { Mean Square } \\
\text { Error(MSE) }\end{array}$ & PSNR \\
\hline $\begin{array}{c}\text { Original } \\
\text { image(JPEG) }\end{array}$ & $188 \mathrm{~KB}$ & - & - \\
\hline TIFF & $1980 \mathrm{~KB}$ & 14.91 & 36.39 \\
\hline JPEG & $188 \mathrm{~KB}$ & 15.09 & 36.34 \\
\hline $\begin{array}{c}\text { DWT - Proposed } \\
\text { Scheme) }\end{array}$ & $1.97 \mathrm{~KB}$ & 24.22 & 19.86 \\
\hline
\end{tabular}

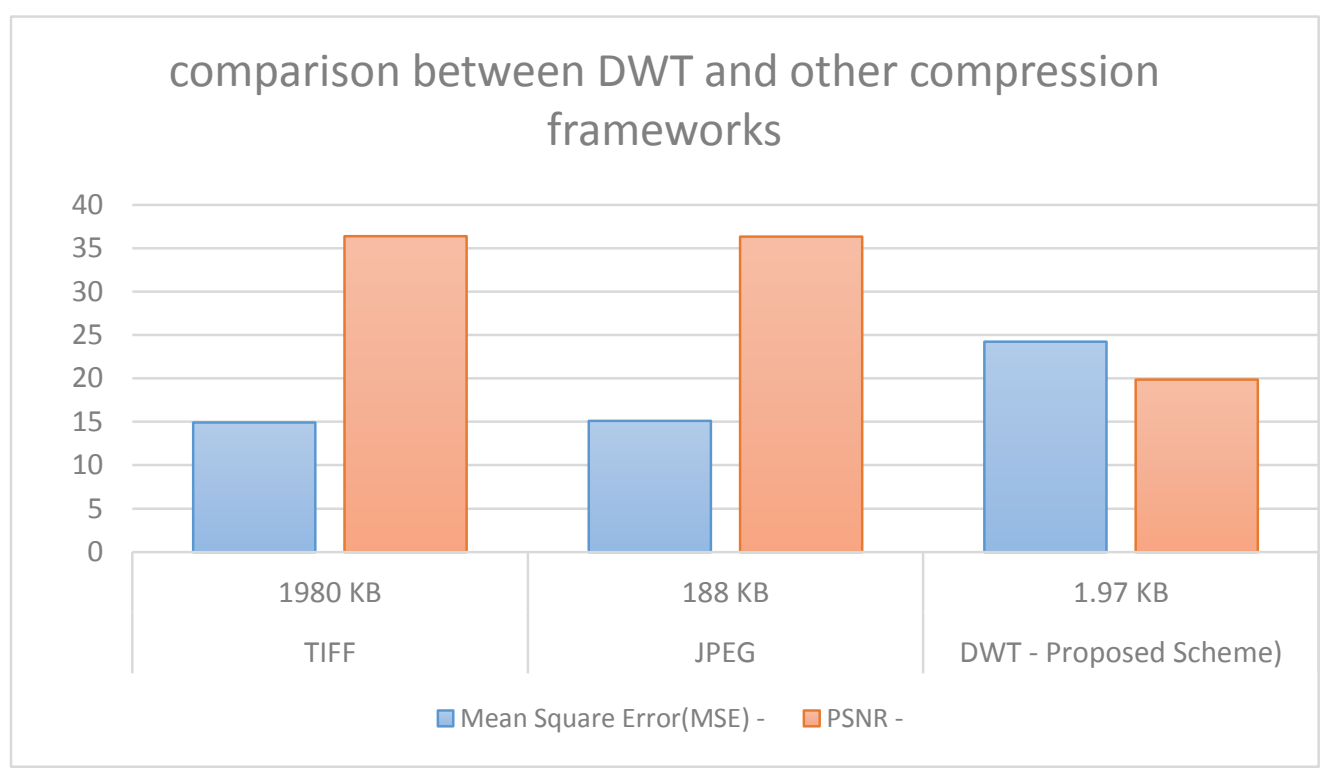

Figure 4-4 Histogram Representation for DWT and other compression frameworks

Table 4-3 Comparison of DWT compression schemes

\begin{tabular}{|l|c|c|c|c|c|}
\hline $\begin{array}{c}\text { DWT } \\
\text { Compression } \\
\text { Types }\end{array}$ & $\begin{array}{c}\text { Original } \\
\text { Image }\end{array}$ & $\begin{array}{c}\text { Compressed } \\
\text { Image }\end{array}$ & $\begin{array}{c}\text { Compression } \\
\text { Ration }\end{array}$ & $\begin{array}{c}\text { Mean } \\
\text { Square } \\
\text { Error }\end{array}$ & PSNR \\
\hline Haar & $92.0 \mathrm{~KB}$ & $70.5 \mathrm{~KB}$ & 1.3050 & 4.85 & 41.27 \\
\hline Bior & $92.0 \mathrm{~KB}$ & $71.3 \mathrm{~KB}$ & 1.2903 & 10.04 & 38.11 \\
\hline Sym & $92.0 \mathrm{~KB}$ & $72.2 \mathrm{~KB}$ & 1.2742 & 8.09 & 39.05 \\
\hline
\end{tabular}




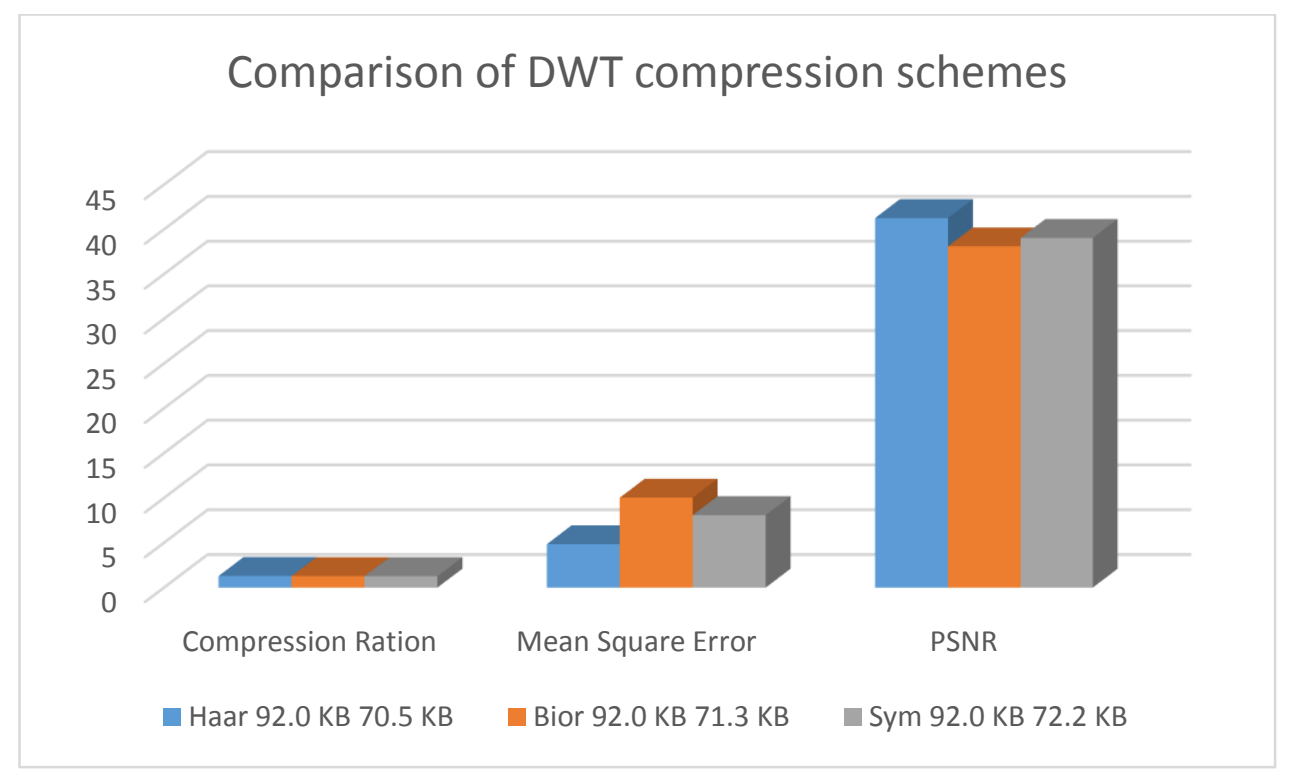

Figure 4-5 Histogram Representation for DWT compression comparison

\subsubsection{Discussion}

Table 4-3 shows results of image sample with resolution $640 \times 640$ and size 92 . $0 \mathrm{~KB}$ in JPEG format for three wavelet types. The wavelet type (sym) proves better compression ration than Haar and Biorthogonal wavelets. But our experiment further tells us that Haar has superior error with peak signal to noise ratio corrections than the two wavelet types.

The experimentation adapted better reconstruction scheme is faster and effective several times than the conventional compression-based algorithms.

\subsection{Image De-noising in Wavelet}

Medical Images often contains lot of artifacts either due to muscle's movement or other electrical interference. De-noising is instrumental in removing artifacts whilst preserving image superiority for fast data relay. Many works is done lately on wavelet thresholding, and image signal de-noising. This is conceivable as wavelet effectively denoise signal with minimal energy compaction with lesser coefficients occurring owing to the artifacts and bigger coefficients because crucial signal features.

\subsubsection{Compression in Wavelet 2D}

This technique uses Matlab GUI wavemenu to remove noise in 2-dimensional digital image. The succeeding phases are performed in de-noising a medical image. 


\begin{tabular}{|c|c|}
\hline$\checkmark$ & Save image to de-noise in a folder and our image was labeled as \\
& "skull_002" JPEG file. \\
\hline$\checkmark$ & Lunch Matlab wavemenu and load the image \\
\hline$\checkmark$ & Select db (2) as wavelet type with level 4 decomposition. \\
\hline$\checkmark$ & Select analyze and click on de-noise \\
\hline$\checkmark$ & Set threshold to default "fixed form threshold". Select hard \\
& threshold. Also set the noise structure to "scaled white noise". \\
& Finally set thresholds by level for horizontal, diagonal and \\
& vertical coefficients. \\
$\checkmark$ & Click on de-noise and finally save your file
\end{tabular}
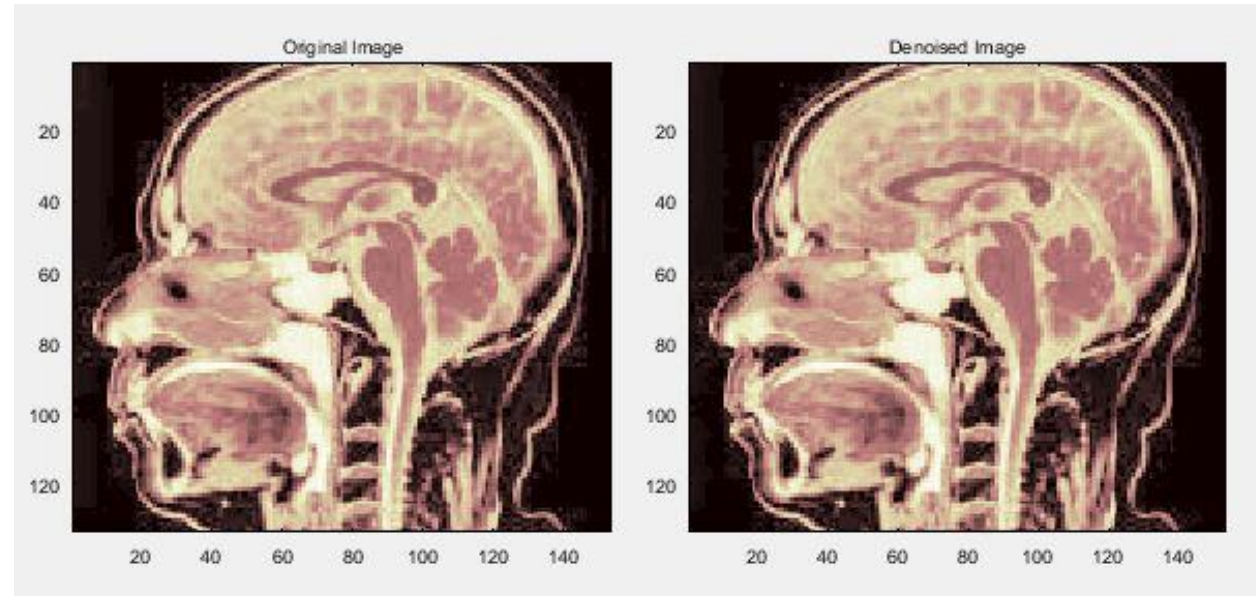

Figure 4-6 (a) DWT de-noised medical image.
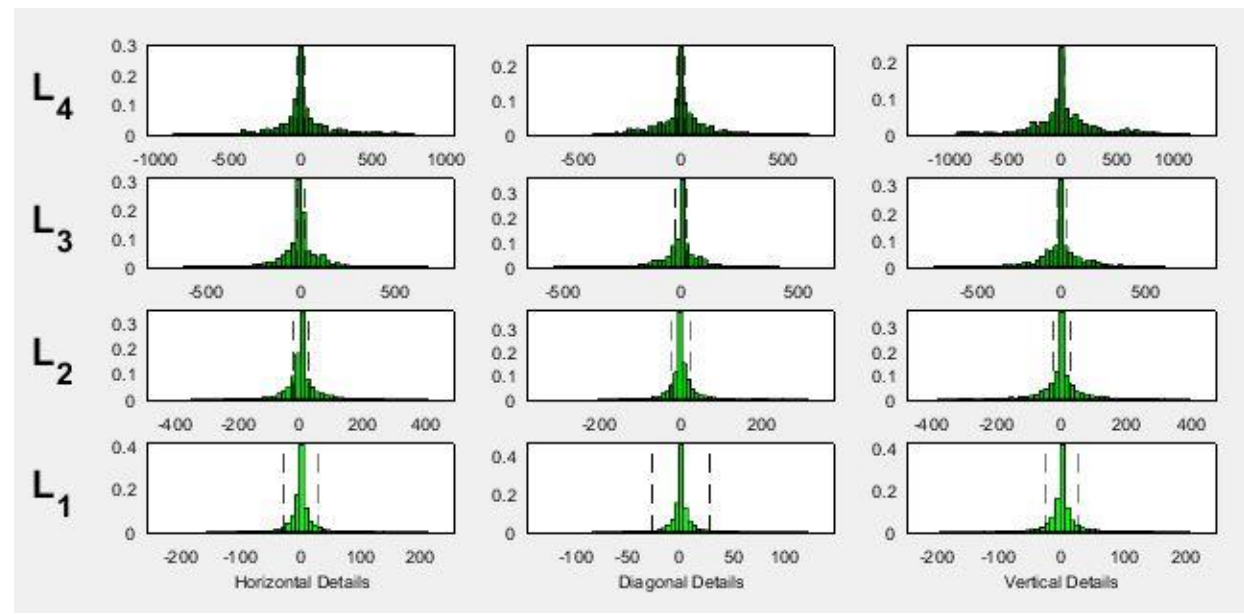

Figure 4-6 (b) four level of the de-noised medical image. 


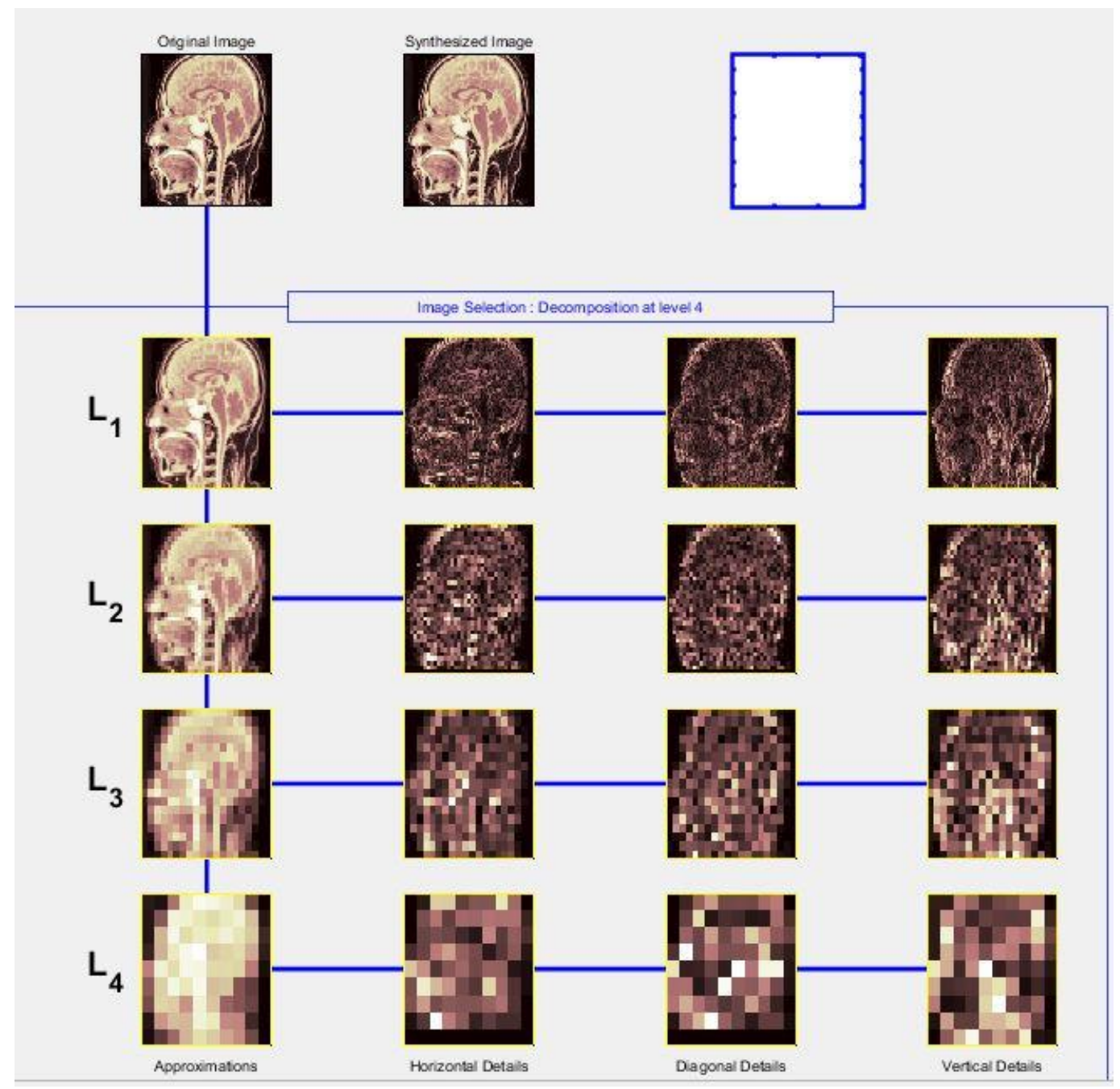

Figure 4-6 (C) More level details of the de-noised medical image.

\subsubsection{DWT De-noising Results}

Our results state the ratio of wavelet coefficients put to zero, and percentage image vitality was conserved in the compression to $99.25 \%$. Here even though our processed image is regained from only half nonzero wavelet details, virtually no noticeable deterioration was present in the image and the quality was preserved.

Table 4-4 Results obtained in figure 4-6.

\begin{tabular}{|c|c|}
\hline DWT Level & Threshold Values \\
\hline Level 1 & 27.49 \\
\hline Level 2 & 25.53 \\
\hline Level 3 & 23.53 \\
\hline Level 4 & 21.62 \\
\hline
\end{tabular}


The table shows that the higher the hard threshold results to lower threshold values using fixed form thresholding method.

\subsection{More DWT Compression Implementation in Matlab}

This technique uses Matlab GUI wavemenu to compress 2 -dimensional digital image. The following procedures are performed in compressing a medicare image with WT wavemenu toolbox ${ }^{[39],[40] ;}$

\begin{tabular}{|cl|}
\hline \multicolumn{2}{|c|}{ Algorithm: Compressing medicare image with WT } \\
\hline$\checkmark$ & Lunch Matlab and load the image \\
\hline$\checkmark$ & Choose coif as wavelet type 1 and select level 3 \\
\hline$\checkmark$ & Save your images \\
\hline
\end{tabular}

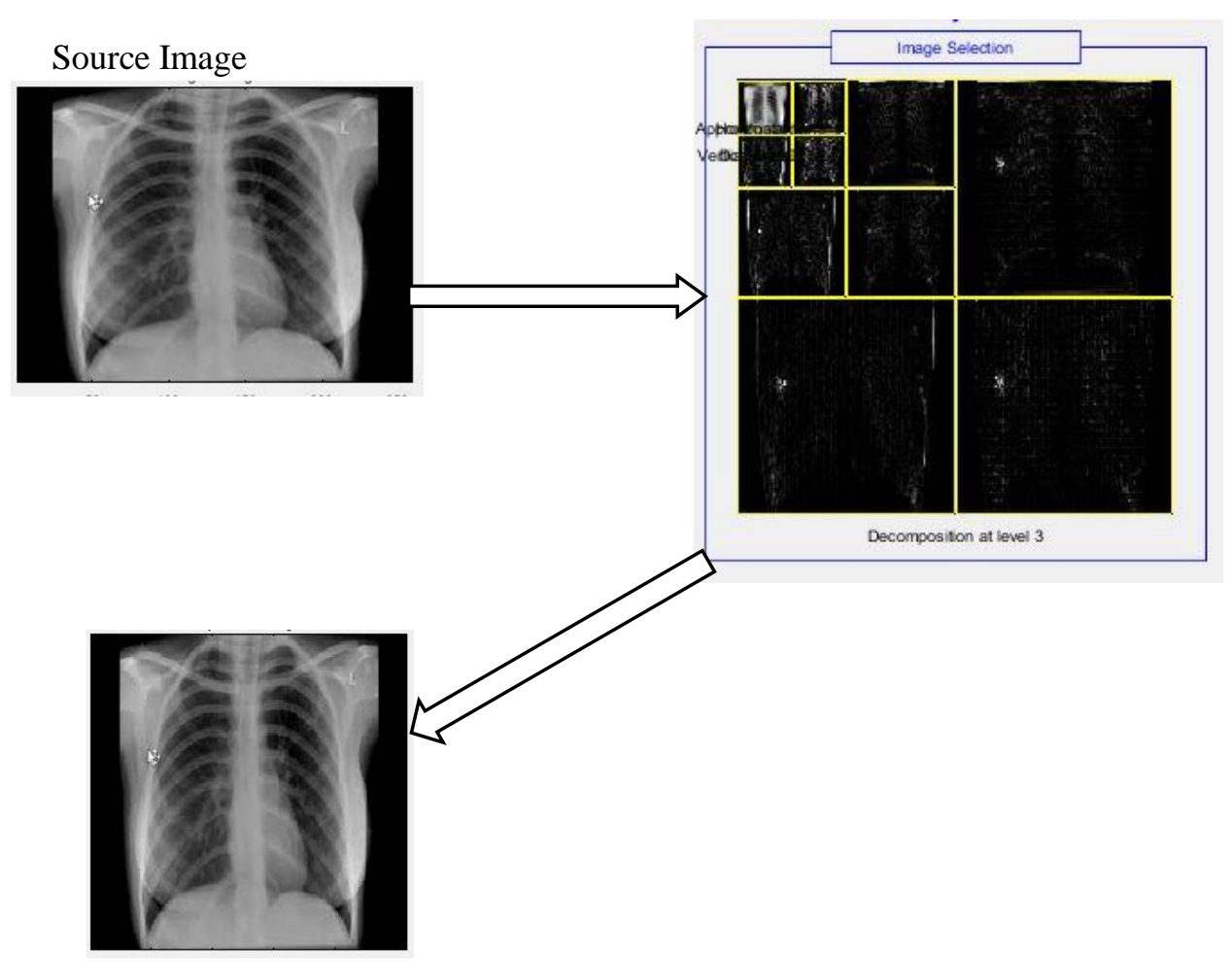

Reconstructed Image

Figure 4-7 Experimental Result done in Matlab

\subsubsection{Experimental Result Discussion for figure 4-7}

A jpg image was compressed applying coif wavelet transform type with size 
16.7KB. After compression, the medical image size was significantly compact to 6.98 $\mathrm{KB}$. This shows that the proposed system is 2 and half times effective than state-of-theart medical image compression-based algorithms. Energy retained was $99.89 \%$ with $93.73 \%$ zero coefficients.

\subsection{Conclusion}

The new structure significantly reduced medical image size and improves hardware design with light-weight devices through wavelet-based algorithms. Moreover, the experiment assesses the efficiency using DWT, Fourier Transform and Fast Fourier Transform frameworks with different wavelet types to compress and de-noise our medical image signal. It was far superior and outperformed other image compressionbased algorithms about 5-times effective. Future direction of our research in compression and de-noising schemes implementing body heat as source of energy 


\section{Chapter 5 Conclusions and Future Recommendations}

\subsection{Introduction}

The section envisages our entire work and evaluates on the conclusions, and future recommendations of our research.

\subsection{Conclusion}

The proposed scheme uses DWT based-compression technique to compress and decompress medical image for reduced transmission times with superior data compressibility. The author was able to achieve his in reducing data storage space. Therefore, our research is directed towards establishment minimal cost, better data compression for analysis of different models resourceful medicare image compressionbased algorithm. Many mother wavelet mother types were used including DWT, Fourier Transform, FFT and Haar to check for high efficiency.

This is necessary because of the rising utilization of health monitoring systems and intelligent agencies as key foundation.

\subsection{Future Work}

Exploring other wavelet-based image compression structures like Daubechies' wavelets is fundamental for future direction. Future work engross enhancing medical image quality by incrementing PSNR value, lowering MSE value and using other metric like percentage root-square distortion(PRD). 


\section{Acknowledgement}

The report would not have been feasible without the divine direction of Allah. I would foremost acknowledge the continuous and relentless supports from my supervisor Professor Jian-Ping $\mathrm{Li}$ in the Computer Science department, School of Computer Science and Engineering of University of Electronic Science and Technology of China for reviewing this piece of work and personal sacrifice in making it a success. I also extend my gratitude to the supervisory committee, Prof. Jian-Ping Li, for their marvelous contributions and insights in fulfilling this work. Worth mentioning Int'l office for awarding me Partial University Scholarship, which I appreciated to the bottom of my heart, and their relentless and supportive efforts during our stay in China. Furthermore, I will also like to thank all lecturers of the Computer Science department and interestingly my lab mates.

I am immensely grateful to Professor Paul Kamara of the Institute of Advanced Management and Technology (IAMTECH) for his sponsorship throughout my education. I deeply thank Mr. Peter Umaru Kamara, Director of Finance at IAMTECH for his endless support towards my education. Thanks to the management and staff of IAMTECH especially Professor Patrick Unisa Taylor for his fatherly advice and moral support and Mr. Ibrahim Sankoh my intimate friend.

I would like to thank Mrs. Elizabeth Ibrahim Guma-Sawaneh for her financial support during my stay in China and Mrs. Isatu Ibrahim Conteh-Sawaneh for her moral support. Special gratitude to all friends and family members especially my mother Fatmata Mansaray, sister Fatmata Sawaneh, brother Lansana Sawaneh, and Dr. Musa Tarawally for their unweaving encouragement and advice during my post graduate struggle. Special thanks and appreciation goes to Dr. Nadir Mustafa Mohammed Osman from Sudan whose help was instrumental in completing this research report. 


\section{References}

[1] Y. Hao and R. Foster, "Wireless body sensor networks for health-monitoring applications," Phys. Meas., vol.29, pp.R27-R56, Nov. 2008.

[2] K. W. Goh, J. Lavanya, Y. Kim, E. K. Tan, and C. B. Soh, "A PDA-based ECG Beat Detector for Home Cardiac care," in IEEE Engineering in Medicine and Biology Society, Shanghai, China, 2005, pp.375-378.

[3] P. Bonato, "Advances in Wearable Technology and Applications in Physical Medicine and Rehabilitation," J. NeuroEng. Rehabil, vol. 2, p. 2, Feb. 2005.

[4] U. Varshney, "Pervasive Healthcare and Wireless Health Monitoring," Mobile Networks and Applications, vol. 12, pp. 113-127, March 2007.

[5] S. Kadambe, R. Murray, G. Paye. Boudreaux-Bartels Wavelet transform-based QRS complex detector, IEEE Transactions on Biomedical Engineering [J]. 1999, 46(7), 838-848

[6] M. J. Weinberger, G. Seroussi and G. Sapiro, "The LOCO-I Lossless Image Compression Algorithm: Principles and Standardization into JPEG-LS", IEEE Trans. On Image Processing, Vol. 2, pp. 1309-1324, Aug. 2000.

[7] Madhuri A. Joshi, "Digital Image Processing, "An Algorithmic Approach", PHI, New Delhi, pp. 175-217, 2006.

[8] S. Bhavani, K. Thanushkodi, "A Survey on Coding Algorithms in Medical Image Compression", International Journal on Computer Science and Engineering, Vol. 02, No. 05, pp. 1429-1434, 2010.

[9] G. K. Kharate, V. H. Pati, "Color Image Compression Based on Wavelet Packet Best Tree”, International Journal of Computer Science, Vol. 7, No. 3, March 2010.

[10] Sachpazidis, Ilias (10 July 2008). "Image and Medical Data Communication Protocols for Telemedicine and Teleradiology (dissertation)" (PDF). Darmstadt, Germany: Department of Computer Science, Technical University of Darmstadt.

[11] Adiloglu, Kamil; Annies, Robert; Wahlen, Elio; Purwins, Hendrik; Obermayer, Klaus (2012). "A Graphical Representation and Dissimilarity Measure for Basic Everyday Sound Events". IEEE Journal of Selected Topics in Signal Processing. 20 (5): 1542-1552. doi: 10.1109/TASL. 2012.2184752.

[12] Scholler, Simon; Purwins, Hendrik (2011). "Sparse Approximations for Drum Sound Classification". IEEE Journal of Selected Topics in Signal Processing. 5 (5): 933-940. doi:10.1109/JSTSP. 2011.2161264.

[13] Lymberis A, Gatzoulis L. Wearable Health Systems: From Smart Technologies to Real Applications. IEEE Engineering in Medicine and Biology Society; New York, NY, USA: 2006. pp. 6789-6792. [PubMed]

[14] Lin G, Tang W. NASA Tech Briefs: Engineering Solutions for Design and Manufacturing. ABP International; New York, NY, USA: 2000. Wearable sensor patches for physiological monitoring; pp. 354-2240. 
[15] Diamond D, Coyle S, Scarmagnani S, Hayes J. Wireless sensor networks and chemo-biosensing. Chem. Rev. 2008; 108:652-679. [PubMed]

[16] Research Europe-Africa Strategy: Strategic Importance of eHealth NEPAD. Accessed 28 January 2016.

[17] E. Jovanov, and D. Raskovic, "Wireless Intelligent Sensors," in R.H. Istepanian, S. Laxminarayan, C.S. Pattichis, Eds, M-Health: Emerging Mobile Health Systems, Springer, 2006.

[18] International Telecommunication Union (ITU) (2005), “ITU Internet Reports 2005: The Internet of Things", ITU, Nov. 2005

[19] B. Kang, F. Liu, Z. Yun, and Y. Liang (2011), "Design of an Internet of Thingsbased smart home system", in Proc. of the 2nd International Conference on Intelligent Control and Information Processing, pp.921-924, 2011

[20] H. Zhang and L. Zhu (2011), "Internet of Things: Key technology, architecture and challenging problems", in Proc. of IEEE International Conference on Computer Science and Automation Engineering, pp.507-512, 2011

[21] Z. Zhang and B. D. Rao, "Extension of SBL algorithms for the recovery of block sparse signals with intra-block correlation," IEEE Trans. on Signal Processing, vol. 61, no. 8, pp. 2009-2015, 2013.

[22] Bailey, David H.; Swarztrauber, Paul N. (1994), "A fast method for the numerical evaluation of continuous Fourier and Laplace transforms", SIAM Journal on Scientific Computing, 15 (5): 1105-1110, doi:10.1787.

[23] Boashash, B., ed. (2003), Time-Frequency Signal Analysis and Processing: A Comprehensive Reference, Oxford: Elsevier Science, ISBN 0-08-044335-4.

[24] J. Walker and T. Nguyen. Wavelet-based image compression [J]. 2001

[25] S. Grgic, M. Grgic, B. Zovko-Cihlar. Performance analysis of image compression using wavelets[J].2001,48(3), 682-695

[26 K. Sayood. Huffman Coding, Introduction. to Data Compression[J]. 2012, 43-89

[27] B B Hubbard, The World According to Wavelets, 2nd edition, Universities Press (India), Hyderabad, 2003.

[28] R M Rao and A S Bopardikar, Wavelet Transforms: Introduction To Theory and Applications, Pearson Education Inc., Delhi, India, 2000.

[29] C S Burrus, R A Gopinath and H Guo, Introduction to Wavelets and Wavelet Transforms - A Pr/mer, Prentice-Hali, New Jersey, USA, 1998.

[30] Liu Bo, Yang Zhaorong, "Image Compression Based on Wavelet Transform", International Conference on Measurement, Information and Control (MIC), 2012

[31] Wang Yannan, Zhang Shudong, Liu Hui, "Study of Image Compression Based on Wavelet Transform”, Fourth International Conference on Intelligent Systems Design and Engineering Applications 2013.

[32] Remya George, Mrs. Manimekalai, "A Novel Approach for Image Compression Using Zero Tree Coding", International Conference on Electronics and Communication System (ICECS -2014), Coimbatore, India

[33] Li, C., Shen, Y., \& Ma, J. (2005). An efficient medical image compression. In 
Engineering I004E Medicine and Biology 27th Annual Conference, 1-4 Sept. 2005. Shangai, China: IEEE.

[34] DL Donoho, De-noising by soft thresholding, IEEE Trans. Inform. Theory, Vol. 41, pp. 613-627, 1995.

[35] Said, A., \& Pearlman, W. A. (to appear). An image multiresolution representation for Lossless and lossy compression. IEEE Transactions on Image Processing.

[36] R. C. Gonzalez, R. E. Woods, S. L. Eddins, —Digital Image Processing using MATLAB\|.

[37] Sonka, M. Hiaual, V. Boyle, R. Image Processing, Analysis and Machine

Vision, 2nd edition. Brooks/Cole Publishing Company.

[38] Jovanov E, Price J, Raskovic D, Kavi K, Martin T, Adhami R. Wireless personal area networks in telemedical environment. Proceedings of the Third International Conference on Information technology in Biomedicine (ITAB-ITIS2000); Arlington, VA, USA. November 2000; pp. 22-27.

[39] Z. Zhang and B. D. Rao, "Extension of SBL algorithms for the recovery of block sparse signals with intra-block correlation," IEEE Trans. on Signal Processing, vol. 61, no. 8, pp. 2009-2015, 2013.

[40] Kanwaljot Singh Sidhu, Baljeet Singh Khaira, Ishpreet Singh Virk, Medical Image Denoising In The Wavelet Domain Using Haar And DB3 Filtering, International Refereed Journal of Engineering and Science (IRJES). 


\section{Appendix I: Calculation of MSE and PSNR in Matlab}

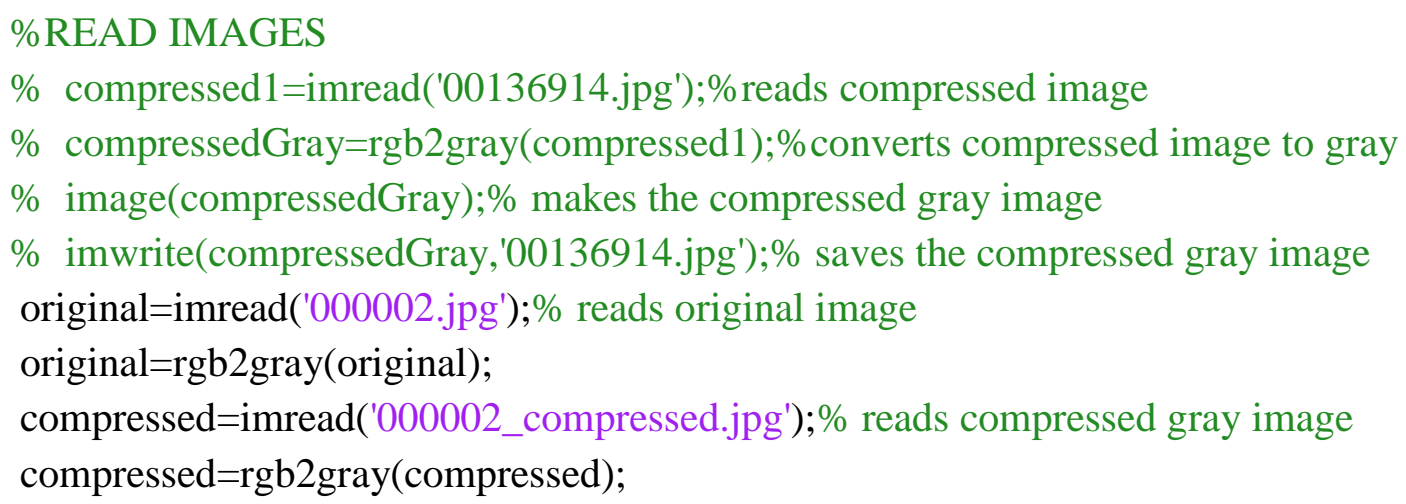




\title{
Appendix II: Fast Fourier Transform implementation code
}

\author{
Step 1: Loading Image \\ clear all, close all, clc \\ \% \\ disp('loading full image ...') \\ $\mathrm{A}=$ imread ('0000101','jpg'); \\ figure (3) \\ imshow $(\mathrm{A})$;
}

Step 2: Coverting the Image to Black Scale

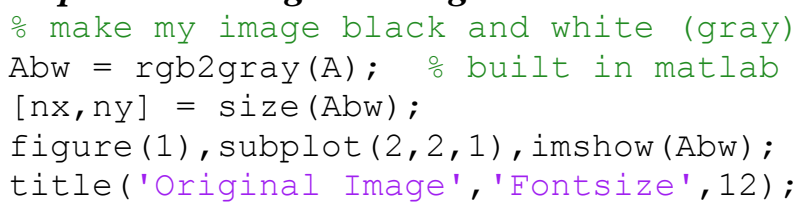

\section{Step 3: Compute the FFT Image}

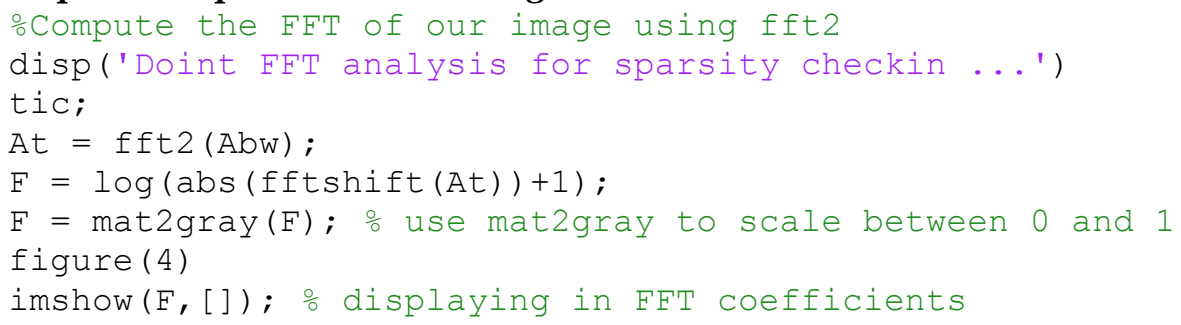

\section{Step 4: Zeroing out Small Coefficients and Inverse Transform}

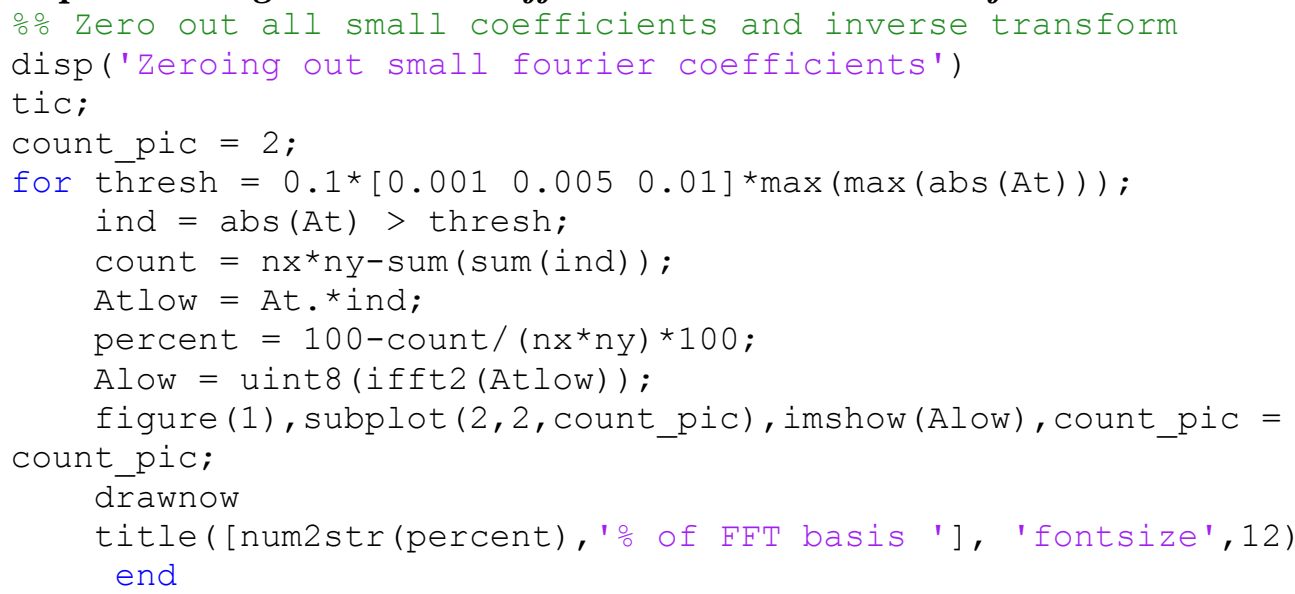

